\title{
BFA1 has multiple positive roles in directing late mitotic events in S. cerevisiae
}

\author{
Whalen $\mathrm{J}^{1 *}$, Sniffen $\mathrm{C}^{2} *$, Gartland $\mathrm{S}^{3}$, Vannini $\mathrm{M}^{2}$, and Seshan $\mathrm{A}^{4}$ \\ *These authors contributed equally.
}

1 Present address: Department of Biology, Tufts University, Medford, MA 02155

2 Present address: Department of Biology, Massachusetts Institute of Technology, Cambridge, MA 02139

3 Present address: Department of Biology, Brandeis University, Waltham, MA 02454

4 Department of Biology, Emmanuel College, Boston, MA 02115

Running title: Bfa1 promotes Tem1 localization to SPBs and initiates efficient cytokinesis in budding yeast

KEYWORDS: Bfa1; Tem1; MEN; mitotic exit; cytokinesis 


\section{ABSTRACT}

The proper regulation of cell cycle transitions is paramount to the maintenance of cellular genome integrity. In budding yeast, the mitotic exit network (MEN) is a Ras-like signaling cascade that effects the transition from M phase to $\mathrm{G} 1$ during the cell division cycle in budding yeast. MEN activation is tightly regulated. It occurs during anaphase and is coupled to mitotic spindle position by the spindle position checkpoint (SPoC). Bfa1 is a key component of the SPoC and functions as part of a two-component GAP complex along with Bub2. The GAP activity of Bfa1-Bub2 keeps the MEN GTPase Tem1 inactive in cells with mispositioned spindles, thereby preventing inappropriate mitotic exit and preserving genome integrity. Interestingly, a GAP-independent role for Bfa1 in mitotic exit regulation has been previously identified. However the nature of this Bub2independent role and its biological significance are not understood. Here we show that Bfa1 also activates the MEN by promoting the localization of Tem1 primarily to the daughter spindle pole body (dSPB). We demonstrate that the overexpression of BFA1 is lethal due to defects in Tem1 localization, which is required for its activity. In addition, our studies demonstrate a Tem1-independent role for Bfa1 in promoting proper cytokinesis. Cells lacking TEM1, in which the essential mitotic exit function is bypassed, exhibit cytokinesis defects. These defects are suppressed by the overexpression of BFA1. We conclude that Bfa1 functions to both inhibit and activate late mitotic events. 


\section{INTRODUCTION}

In S. cerevisiae, the inactivation of the cyclin B homolog $\mathrm{Clb} 2$ triggers the transition from mitosis into $\mathrm{G} 1$ known as exit from mitosis. This process is regulated by a small-guanine nucleoside triphosphatase (GTPase) signaling cascade known as the mitotic exit network (MEN; Stegmeier and Amon, 2004). Tem1, a small GTPase localized to spindle pole bodies (SPBs), functions at the top of the pathway and activates the downstream Hippo-like kinase Cdc15 (Bardin et al., 2000; Pereira et al., 2000; Asakawa et al., 2001; Lee et al., 2001; Visintin and Amon, 2001). Cdc15 is coordinately recruited to SPBs and activated by both Tem 1 and by the Polo protein kinase Cdc5 (Cenamor et al., 1999; Bardin et al., 2000; Pereira et al., 2000; Xu et al., 2000; Molk et al., 2004; Rock and Amon, 2011). Cdc15 in turn activates the Ndr-family kinase Dbf2 (Mah et al., 2001; Visintin and Amon, 2001). The activation of Dbf2 requires an associated factor, Mob1, and is preceded by the recruitment of Mob1 to the MEN scaffold Nud1 at SPBs (Komarnitsky et al., 1998; Luca and Winey, 1998; Mah et al., 2001; Rock et al., 2013). The activation of Mob1-Dbf2 at the SPB and subsequent dissociation of this complex from SPBs results in the activation of the Cdc14 phosphatase, the ultimate effector of the pathway. Cdc14 is held inactive in the nucleolus by its inhibitor Cfi1/Net1. The Mob1-Dbf2 complex causes the release of Cdc14 from Cfi1 during late anaphase, which allows Cdc14 to spread throughout the nucleus and cytoplasm and reach its targets. 
Activated Cdc14 triggers mitotic CDK inactivation and hence exit from mitosis (Jaspersen et al., 1999; Shou et al., 1999; Visintin et al., 1999).

The regulation of Tem1 activity is important for the integrity of two cell cycle checkpoints: the Spindle Assembly Checkpoint (SAC) and the Spindle Position Checkpoint (SPoC). The SAC acts in metaphase to monitor mitotic spindlechromosomal attachments before anaphase onset, thereby preventing chromosome missegregation and aneuploidy (reviewed in Musacchio and Salmon, 2007). The SPoC acts in anaphase to monitor alignment of the segregating chromosomes along the mother-bud axis (reviewed in Caydasi et al., 2010). A key role of the two component GTPase activating (GAP) complex Bub2-Bfa1 is to restrain Tem1 activation in the presence of unattached chromosomes or in the case of mitotic spindle misalignment (Alexandru et al., 1999; Fesquet et al., 1999; Fraschini et al., 1999). This, in turn, prevents unscheduled mitotic exit. Indeed, increased levels and activation of Tem1 lead to an increased proportion of cells that bypass the SAC (Chan and Amon, 2009).

Therefore it is important to have a thorough understanding of how Tem 1 activation is controlled.

The recruitment of Tem 1 to SPBs is crucial for its activity. For example, a strain in which Tem 1 is mis-localized to the plasma membrane is unable to exit from mitosis (Valerio-Santiago and Monje-Casas, 2011). Conversely, a fusion between Tem1 and the SPB outer plaque component Cnm67 (Tem1-Cnm67) localizes constitutively to both SPBs and leads to bypass of the SPoC (Valerio-Santiago and Monje-Casas, 2011). Thus, Tem1 localization is intertwined with its activation, but how is this process regulated? 
Like Tem1, both Bub2 and Bfa1 also localize to SPBs. Bub2 localization is dependent upon Bfa1 localization and Bfa1 is asymmetrically localized to the dSPB from early anaphase through cytokinesis (Pereira et al., 2000). There is mounting evidence that Tem1 and Bfa1 localization to SPBs is interdependent. For example, in cells lacking BFA1, Tem1 localizes to a much lesser extent to both SPBs from metaphase to late anaphase, but is clearly present on both SPBs in telophase. However, this does not impair the ability of cells to exit from mitosis in a timely manner (Pereira et al., 2000; Valerio-Santiago and Monje-Casas, 2011). Tem1 localization influences the residence of Bfa1 on the SPBs, although Bfa1 can localize to the dSPB in the absence of Tem1 function (Pereira et al., 2000). In the presence of a Tem1-Cnm67 fusion, Bfa1 is found symmetrically on both SPBs in metaphase and anaphase cells (Valerio-Santiago and Monje-Casas, 2011). Lastly, tethering Bfa1 to the SPBs constitutively using a SPC72-BFA1 fusion leads to partial bypass of the SPoC and suppresses the localization-defective tem1-3 allele (Scarfone et al., 2015). These results provide evidence that Bfa1 may have a positive effect on Tem1 localization and therefore, on MEN activation. However, such a role has not been clearly explored thus far.

A MEN-promoting function for Bfa1 would need to be a GAP-independent function. Interestingly, a GAP-independent role for Bfa1 in mitotic exit has been previously described. Bub2 and Bfa1 together increase the intrinsic GAP-activity of Tem1 in vitro. Paradoxically, Bfa1 alone appears to inhibit both GTP dissociation and GTP hydrolysis of Tem1 in vitro, while having no affect on GDP dissociation (Geymonat et al., 2002). These in vitro data would suggest that Bfa1 positively regulates Tem1, once it 
becomes GTP-bound, and predict that Bfa1, when overexpressed, could activate the MEN. However, the overexpression of BFA1 instead produces a cell cycle block in anaphase. Interestingly, Ro et al. showed that this terminal arrest was independent of $B U B 2$, suggesting that a GAP-independent function of BFA1 causes the arrest (Ro et al., 2002). Taken together, these data imply that Bfa1 primarily has a negative role in the regulation of Tem 1 in vivo. However, the BUB2-independent function of BFA1 on MEN regulation is unknown.

In this study we demonstrate that BFA1 overexpression using a GAL-BFA1 allele leads to a defect in Cdc14 activation. We find that this defect stems from an inability of Tem1 to localize correctly to SPBs in the presence of GAL-BFA1. Further, we show that the GAL-BFA1 mitotic exit defect is completely suppressed by co-overexpression of TEM1. We confirm that the overexpression of BFA1 does not affect Mob1-Dbf2 activation during mitotic exit. Interestingly, our data also suggest that Bfa1 may have a MEN-dependent positive function during cytokinesis. These data underscore the positive role for Bfa1 on Tem1 localization and activation, and suggest a novel role for Bfa1 in promoting efficient cytokinesis.

\section{RESULTS}

\section{The overexpression of BFA1 causes a defect in Cdc14 activation.}

Previous studies characterizing the effects of the GAL-BFA1 allele on mitotic exit demonstrated that these cells delay in anaphase upon galactose induction. 
Furthermore, it was known that this severe anaphase delay was not dependent on $B U B 2$

(Li, 1999; Ro et al., 2002; Figure 1). In order to elucidate the nature of the BUB2-

independent effects of BFA1 on anaphase progression, we first sought to determine whether overexpression of BFA1 affects the release of Cdc14 from its inhibitor Cfi1. In wild type cells undergoing a synchronous cell cycle, the timing of anaphase correlates with the full (nuclear and cytoplasmic) release of Cdc14 from the nucleolus, where it is held inactive by its inhibitor Cfi1. This event is a marker for MEN activation (Jaspersen et al., 1999; Shou et al., 1999; Visintin et al., 1999). We arrested cells in G1 using alphafactor pheromone and released them into a synchronous cell cycle. We monitored the appearance and disappearance of metaphase and anaphase spindles, as well as Cdc14 localization. Specifically, we analyzed the localization of Cdc14 in anaphase cells. We found that synchronized GAL-BFA1 cells exhibit a severe anaphase delay and also fail to exhibit full Cdc14 activation and release to the nucleus and cytoplasm (Figure 2A).

We next examined whether the deletion of CFI1 would ameliorate the anaphase delay of GAL-BFA1 cells. While the induction of BFA1 overexpression in GAL-BFA1 cells led to a significant anaphase delay, GAL-BFA1 cfi1 $\Delta$ cells resembled wild type cells and did not have anaphase defects (Figure 2B). Taken together, these results demonstrate that Cdc14 activation is blocked by GAL-BFA1 and that the effects of BFA1 overexpression can be attenuated by Cdc14 hyperactivation.

\section{BFA1 overexpression causes de-localization of Tem1 from SPBs.}


We were interested in determining the specific step in the MEN that was perturbed by the GAL-BFA1 allele. There is prior evidence that Tem1 and Bfa1 localization to SPBs is at least partially interdependent (Pereira et al., 2000; ValerioSantiago and Monje-Casas, 2011; Scarfone et al., 2015). Therefore, we hypothesized that both Tem1 and Bfa1 localization were impaired when BFA1 was overexpressed. We first examined the localization of the GAL-GFP-BFA1 allele. We examined anaphase cells and were surprised to find that in the presence of galactose, Bfa1-GFP is concentrated at the dSPB, as it is in wild type cells (Pereira et al., 2000). However, we also noted that significant cytoplasmic signal is present in these cells (Figure 3A).

Given the significant cytoplasmic localization of Bfa1 in GAL-BFA1 cells, we reasoned that Tem1 could be dragged off of the SPB and into the cytoplasm in these cells. Indeed we found that Tem1-GFP was delocalized from SPBs in $100 \%$ of cells when BFA1 was overexpressed by galactose addition. In contrast, when these same cells were cultured in the presence of raffinose alone, a majority of the cells had Tem1 localized to one or two SPBs (Figure 3). These results demonstrate that Tem1 localization is defective in GAL-BFA1 cells.

\section{Tem1 re-localization to SPBs does not fully suppress GAL-BFA1 mitotic exit defects.}

Now that we had determined that Tem1 localization is affected in GAL-BFA1 cells, we hypothesized that we could suppress this defect by artificially re-localizing Tem1 to SPBs in BFA1 overexpressing cells. In the presence of a Tem1-Cnm67 fusion, Bfa1 is found symmetrically on both SPBs in metaphase and anaphase cells (Valerio- 
Santiago and Monje-Casas, 2011). Therefore we analyzed the kinetics of Cdc14 release from Cfi1 in the nucleolus and the kinetics of mitotic exit in GAL-BFA1 TEM1-eGFPCNM67 cells. In this experiment, we analyzed the localization of Cdc14 in anaphase cells to the nucleolus, the nucleus, and the nucleus and cytoplasm. This allowed us to distinguish between cells with inactive Cdc14 (sequestered in the nucleolus), Cdc14 released by the Cdc Fourteen Early Anaphase Release (FEAR) network (nucleus only; reviewed in Rock and Amon 2009), and Cdc14 released in late anaphase by the MEN into the nucleus and the cytoplasm. We found that the partial release of Cdc14 to the nucleus that occurs in early anaphase due to FEAR pathway activity was unaffected in GAL-BFA1 cells. However, as shown previously these cells accumulated in anaphase and $63 \%$ of anaphase cells displayed Cdc14 sequestration in the nucleolus, indicating that the MEN was inhibited in these cells (Figure 4F). We found that cells containing both GAL-BFA1 and TEM1-eGFP-CNM67 were able to exit from mitosis in a slightly timelier manner than GAL-BFA1 cells, though they were by no means able to progress like wild type cells (Figure 4). However, we observed only a partial suppression of the anaphase delay, as $31 \%$ of anaphase cells in the GAL-BFA1 TEM1-eGFP-CNM67 background still had Cdc14 sequestered in the nucleolus (Figure 4F). Importantly this partial suppression was not due to de-localization of Tem1-Cnm67 in the GAL-BFA1 background, as the Tem1-eGFP-Cnm67 protein was observed at the dSPB or at both mother and daughter SPBS regardless of GAL-BFA1 induction. Interestingly, we observed that in the presence of the GAL-BFA1 allele, a larger fraction of anaphase cells exhibited a bias for Tem1- 
Cnm67 dSPB localization than in TEM1-CNM67 cells that did not overexpress BFA1, which further suggests that Bfa1 influences Tem1 localization to SPBs (Figure 4G-H).

GAL-TEM1, but not TEM1-2 $\mu$, can fully suppress GAL-BFA1 mitotic exit defects.

We considered two hypotheses for the partial suppression of GAL-BFA1 by TEM1-CNM67. One possibility was that the TEM1-CNM67 allele provides low levels of activated Tem 1 and higher levels are needed to fully bypass GAL-BFA1. A second, nonmutually exclusive possibility was that GAL-BFA1 has TEM1-independent effects on MEN activation. We decided to test this first possibility by examining the suppression of GALBFA1 by two different alleles of TEM1: TEM1-2 $\mu$ and GAL-TEM1. We predicted that TEM1-2 $\mu$ would also exhibit a partial bypass of GAL-BFA1 because this allele also has lower levels of activated Tem1. Conversely, we expected to observe complete suppression of the effects of GAL-BFA1 by the GAL-TEM1 allele because this allele exhibits high levels of activated Tem1 (Chan and Amon, 2009). We synchronized cells and utilized spindle and nuclear morphology were used to assess the timing of metaphase, anaphase and mitotic exit. We found that the anaphase delay phenotype observed in GAL-BFA1 was not suppressed in GAL-BFA1 TEM1-2 $\mu$ cells, which contained the TEM1 gene on a multicopy plasmid (Figure 5). In contrast, the timing of mitotic exit in GAL-BFA1 GAL-TEM1 cells overexpressing both BFA1 and TEM1 was indistinguishable from wild type cells (Figure 6). We noted that the extent of the anaphase delay observed in GAL-BFA1 cells varied depending upon the type of media utilized and the temperature at which cells were cultured (compare 4B, 5B, and 6B). However regardless 
of culturing conditions, these data support the hypothesis that defects in Tem 1 activation are the primary reason that GAL-BFA1 cells cannot activate the MEN.

\section{Dbf2 localization to the budneck is enhanced by GAL-BFA1.}

Our prior data strongly suggested that the mislocalization and faulty activation of Tem1 in GAL-BFA1 cells resulted in a failure to activate the MEN. However, in order to confirm that the GAL-BFA1 allele did not impart any TEM1- independent mitotic exit defects, we sought to analyze MEN activation in GAL-BFA1 cells that lacked Tem1 activity. The deletion of TEM1 confers a lethal phenotype. In order to keep tem $1 \Delta$ cells alive, the hyperactive CDC15-UP allele, in which CDC15 is overexpressed using a GALinducible promoter, was utilized (Rock and Amon, 2011). We examined the localization of Dbf2-eGFP to SPBs as a proxy for MEN activation in tem1 $\triangle$ CDC15-UP cells. Dbf2 is known to localize to SPBs during late anaphase following Cdc15 activation, but the protein also localizes to the budneck where it activates the process of cytokinesis (Stegmeier and Amon, 2004; Frenz et al., 2000; Meitinger et al., 2010; Oh et al., 2012; Meitinger et al., 2013). In order to fully assess the effects of GAL-BFA1 on Dbf2 localization, we quantified the number of anaphase cells with Dbf2-eGFP at the SPBs, at the budneck, at both of these structures, and at neither of these structures (Figure 7A). In large budded wild type cells containing one Spc42-mCherry SPB dot distributed in mother and daughter cells, Dbf2-eGFP is found at the SPBs in $42.8 \%$ of cells (SPB only + SPB and budneck; Figure 7A). In tem1 $\triangle$ CDC15-UP and tem1 $\triangle$ CDC15-UP GAL-BFA1 cells 
Dbf2-eGFP is found at SPBs in $57.6 \%$ and $73.3 \%$ of cells respectively (SPB only + SPB and Budneck; Figure 7A). These data indicate that the overexpression of BFA1 does not have TEM1-independent mitotic exit effects.

Unexpectedly, when we examined the budneck localization of Dbf2-eGFP in these same cells, we found that Dbf2-eGFP budneck localization is impaired in tem1 $\Delta$ CDC15-UP anaphase cells. Specifically, wild type cells exhibit budneck localization in $43.7 \%$ of cells, while tem1 $\triangle$ CDC15-UP cells only have Dbf2-eGFP at the budneck in $26.4 \%$ of cells (Budneck only + SPB and Budneck; Figure 7A). In tem1 $\triangle$ CDC15-UP GALBFA1 cells, however, the fraction of anaphase cells with Dbf2-eGFP localized to the budneck is returned to $45.8 \%$ (Budneck only + SPB and Budneck; Figure 7A). These data indicated that, despite the fact that tem1 $\triangle C D C 15-U P$ cells display normal cell cycle kinetics and Dbf2 kinase activity during anaphase (Rock and Amon, 2011), the cytokinesis-specific role of Dbf2 was disrupted. They also suggested that the overexpression of BFA1 could suppress these defects in tem1 $\triangle$ CDC15-UP cells.

To further explore whether cytokinesis defects were present in tem1 $\triangle$ CDC15-UP cells, and to determine whether the deletion of TEM1 or the overexpression of CDC15 were responsible for these defects, we analyzed the cellular morphology of these cells following a brief sonication. This allows the separation of cell clumps without disrupting cell walls. We found that less than $2 \%$ of wild type and CDC15-UP cells exhibited cytokinesis defects where two or more cell bodies remained connected after brief sonication (Figure $7 \mathrm{~B}, 7 \mathrm{C}$ ). In contrast, $31 \%$ of tem $1 \triangle \mathrm{CDC} 15-\mathrm{UP}$ cells exhibited chained cell morphology where two or three cell bodies remained connected (Figure 7B, 7D). 
Furthermore, we observed that the cytokinesis defects of tem1 $\triangle C D C 15-U P$ cells were largely suppressed in the presence of GAL-BFA1. In tem1 $\triangle$ CDC15-UP GAL-GFP-BFA1 cells, only $5.5 \%$ of cells exhibited chains (Figure 7B, 7E). These data imply that increased levels of BFA1 promote cytokinesis by a Tem1-independent mechanism.

\section{DISCUSSION}

The activation of the MEN is essential for the destruction of mitotic CDK activity at the M phase to $\mathrm{G} 1$ transition. Several components of the MEN, as well as the MEN regulators Bfa1 and Bub2, localize to either one or both SPBs (reviewed in Stegmeier and Amon, 2004). Furthermore, mutations or conditions that perturb SPB localization of Tem1, Cdc15, Mob1, and Dbf2 have been shown to affect timely mitotic exit as well as mitotic checkpoint functions (reviewed in Musacchio and Salmon, 2007; reviewed in Scarfone and Piatti, 2015). Therefore, it is important to deepen our understanding of how the localization of these components to SPBs is regulated. Our findings highlight a positive role for Bfa1 in the localization of Tem1, which is required for MEN activation. In addition, we show that Bfa1 promotes efficient cytokinesis by an unknown mechanism. These data provide another example of how the temporal coupling between mitotic exit and cytokinesis is established in budding yeast.

\section{BFA1 overexpression inhibits MEN activity but not FEAR activity.}

The well-established role of Bfa1 is to negatively regulate the MEN by 
stimulating Tem1's GTPase activity in complex with the enzymatic component Bub2 (Pereira et al., 2000; Geymonat et al., 2003). However, increasing cellular Bfa1 protein levels using a GAL-BFA1 allele prevents mitotic exit even in the absence of Bub2 (Ro et al., 2002 and this study). We showed that in GAL-BFA1 cells there is no MEN-induced release of Cdc14 observed and the cells delay in anaphase. The anaphase delay phenotype is suppressed by hyperactivation of Cdc14 using the $c f i 1 \Delta$ allele (Shou et al., 1999; Visintin et al., 1999; Figure 2B). These results confirm that there is a Bub2independent role for Bfa1 in the regulation of the MEN, which functions to promote Cdc14 release from the nucleolus.

The anaphase delay phenotype in GAL-BFA1 cells was variable depending upon temperature. For example, we observed that in rich media at $25^{\circ} \mathrm{C}, 100 \%$ of cells arrested anaphase (Figure 6B). However, at $21^{\circ} \mathrm{C}$ in rich media, GAL-BFA1 cells exhibit a 40 minute delay in anaphase with $63 \%$ of anaphase cells containing Cdc14 sequestered in the nucleolus, but eventually these cells break down their spindles (Figure 4B). It is not clear why the phenotype is variable, but we speculate that the overexpression of BFA1 is less severe in cells that are growing more slowly and therefore have a prolonged metaphase. We noted that the FEAR-mediated release of Cdc14 was not affected by GAL-BFA1 (Figure 4F). It is possible that cell-to-cell differences in FEAR network component levels and activity produced especially high levels of FEAR-dependent Cdc14 activation, which allowed some anaphase cells to exit from mitosis despite high levels of MEN-inhibition by GAL-BFA1. A FEAR-dependent mitotic exit despite MEN-inhibition has been previously shown in cells with mispositioned anaphase spindles. Falk et al. 
demonstrated that a small number of otherwise wild type cells exit from mitosis inappropriately, even in the presence of mispositioned spindles. However, this inappropriate mitotic exit is completely prevented in cells where the FEAR network has been inactivated by the deletion of the FEAR components SPO12 or SLK19 (Falk et al., 2016). If FEAR activity were to be a prerequisite for a proportion of GAL-BFA1 anaphase cells growing at $21^{\circ} \mathrm{C}$ to exit from mitosis, the deletion of SPO12 in these cells would be predicted to extend the anaphase delay.

\section{BFA1 overexpression does not inhibit MEN through symmetric SPB localization.}

Bfa1, when present in the cell at endogenous levels, localizes preferentially to the dSPB (Pereira et al., 2000). When the protein is overexpressed, we see that a significant proportion of GFP-Bfa1 is present at the dSPB and also in the cytoplasm (Figure 3A). Previous studies have suggested that a shift from symmetric Bfa1-Bub2 SPB localization to asymmetric localization pattern precedes the activation of Tem1 and mitotic exit (Pereira et al., 2000, 2001; Molk et al., 2004; Fraschini et al., 2006; Maekawa et al., 2007; Geymonat et al., 2009; Kim et al., 2012). In addition, Cdc5 inactivates Bfa1 in anaphase by phosphorylating Bfa1 (Hu et al., 2001; Geymonat et al., 2003). Therefore, the overexpression of BFA1 could potentially overwhelm the Cdc5-dependent inhibitory phosphorylation of the protein, and thereby contribute to Bfa1 hyperactivity. However, our finding that GFP-Bfa1 localized asymmetrically to the dSPB in GAL-BFA1 cells argues against this, as the Bfa1-4A mutant, in which the four Cdc5 phosphorylation sites in Bfa1 are mutated, displays a symmetric localization pattern (Kim et al., 2012). This 
observation further suggested that the block in MEN activation in the presence of increased Bfa1 protein was not due to increased Bfa1 inhibitory activity.

\section{Bfa1 promotes Tem1 SPB localization.}

Several lines of evidence indicate that BFA1 overexpression deters mitotic exit by preventing proper localization of Tem1 to the dSPB. First, we show that Tem1 is delocalized from SPBs in GAL-BFA1 strains (Figure 3B, 3C). Tem1 localization to SPBs is likely to be a pre-requisite for mitotic exit. Tethering the protein to the plasma membrane using a Tem1-CAAX fusion blocks mitotic exit. On the other hand, the fusion of TEM1 to CNM67, which encodes a component of the outer plaque of the SPB, causes mitotic to occur even in cells that have mispositioned anaphase spindles (ValerioSantiago and Monje-Casas, 2011). We demonstrated that artificially tethering Tem1 to SPBs constitutively using the TEM1-CNM67 allele leads to a partial suppression of GALBFA1 lethality (Figure 4). This finding confirms that removal of Tem1 from the SPBs is a key mechanism by which the overexpression of BFA1 blocks mitotic exit and argues for a positive function for Bfa1 in mitotic exit regulation.

Although mitotic exit did proceed to some extent in GAL-BFA1 TEM1-CNM67 cells, a significant proportion of anaphase cells (31\%) still had Cdc14 sequestered in the nucleolus (Figure 4E). We propose that this is due to the weak allele strength of the TEM1-CNM67 allele. Using a checkpoint bypass assay, Chan and Amon previously determined that hyperactive alleles of TEM1 confer differing levels of MEN hyperactivation. Specifically, the GAL-TEM1 allele produced the highest MEN activation 
level in this assay, while both TEM1-CNM67 and TEM1-2micron alleles produced lower levels of MEN activation (Chan and Amon, 2009). In keeping with this, we observed that only the presence of GAL-TEM1 completely abolished GAL-BFA1 inhibition of mitotic exit (Figure 6). In addition, we found that GAL-BFA1 posed no impact on the SPB localization of the downstream MEN kinase Dbf2 when the effects on Tem1 were bypassed. Specifically, cells lacking TEM1 and kept alive using a hyperactive CDC15-UP allele showed normal recruitment of Dbf2 to SPBs both in the presence and absence of overexpressed BFA1 (Figure 7A). Together, these results support the conclusion that Tem1 mis-localization is the central mitotic exit defect in GAL-BFA1 cells.

Why is does the TEM1-CNM67 allele display lower levels of activity, despite the fact that Tem1 localization to SPBs is a prerequisite for its activation? One reason could be that the Tem1-Cnm67 fusion at the SPBs is subject to increased GAP activity, which prevents the protein from being highly active. While we acknowledge that this is possible, we do not favor this explanation because TEM1-CNM67 bfa1 $\Delta$ cells, in which the GAP is inactivated, display the same SPoC bypass phenotype as TEM1-CNM67 cells (Valerio-Santiago and Monje-Casas, 2011). These data indicate that GAP inactivation does not enhance the activity of TEM1-CNM67 allele.

The TEM1-CNM67 allele displays a significantly more symmetric SPB localization than the wild type TEM1, and causes endogenous Bfa1 protein to localize in a symmetric manner (Valerio-Santiago and Monje-Casas, 2011; Figure 4G). Here, we showed that when BFA1 is overexpressed, Bfa1, which is concentrated in high amounts at the dSPB, led to a largely asymmetric localization of the Tem1-Cnm67 chimeric protein to the dSPB 
(Figure 4G). This confirms that Tem1-Cnm67 and Bfa1 interact at the SPB even when Bfa1 is present at high levels. This also provides further evidence that Bfa1 acts as a receptor for Tem1 at the dSPB.

\section{How does overexpressed BFA1 disrupt Tem1 localization to SPBs?}

Tem1 requires Bfa1 for efficient loading onto SPBs, though the protein can be found on SPBs at low levels even in the absence of BFA1 (Pereira et al. 2000; ValerioSantiago and Monje-Casas 2011). Although it is not known exactly how Tem1 associates with SPBs, Tem1 itself can associate with both Bfa1 and Nud1 in two-hybrid binding assays (Kim et al., 2008; Valerio-Santiago and Monje-Casas, 2011). Bfa1 itself binds in a complex at the SPB outer plaque with both Nud1 and Spc72 (Gruneberg et al., 2000; Gryaznova et al., 2016). It has been suggested that in the absence of BFA1, Tem1 may bind to Nud1 (Valerio-Santiago and Monje-Casas, 2011). Therefore, it is possible that the large amounts of Bfa1 that are present saturate the outer plaque, and specifically Nud1, and prevent the localization of Tem1 to this organelle. However, we favor the idea that the strong association between cytoplasmic Bfa1 in the GAL-BFA1 allele and Tem1 titrates Tem1 away from the SPB. Notably, in tem1 $C D C 15-U P$ GAL-BFA1 cells, Dbf2eGFP is observed at SPBs, presumably bound to its receptor phospho-Nud1 (Rock et al., 2013; Figure 7). This indicates that increased levels of Bfa1 on the dSPB do not prevent the association of other MEN components with the Nud1 scaffold on the outer plaque. 


\section{Bfa1 serves as both an activator and an inhibitor of late $M$ phase events.}

The essential mitotic exit function of Tem1 is to recruit Cdc15 to the SPB by an unknown mechanism (Rock et al., 2011). Previous work has demonstrated that the fusion of BFA1 with TEM1 or with the SPB outer plaque component SPC72 leads to increased, rather than decreased, MEN activation by increasing the residence time of Tem1 at SPBs (Valerio-Santiago and Monje-Casas, 2011; Scarfone et al., 2015). Our results are consistent with the notion that Bfa1 can enhance Tem1 activity and MEN activation by bringing Tem 1 to the SPB, and that preventing Tem1 localization by toggling the cellular levels of Bfa1 can conversely inhibit MEN.

The Septation Initiation Network (SIN) in S. pombe is homologous to the MEN, but controls septation and cytokinesis rather than mitotic cyclin inactivation (reviewed in Krapp and Simanis, 2008). The Tem1 homolog Spg1p and the Bfa1 homolog Byr4p also localize to the SPB in an asymmetric manner (Cerutti and Simanis, 1999; Li et al., 2000). Byr4p serves as a bridge on the SPB between Spg1p and the Bub2 homolog Cdc16p, which is the enzymatic component of the Byr4p-Cdc16p two-component GAP complex (Furge et al., 1998). It was previously shown that overexpression of Byr4p blocks SIN activation and cytokinesis due to the titration of the Spg1p GTPase off the SPB (Li et al. 2000). This suggests that the cellular levels of both Bfa1 and Byr4p could similarly be important to ensure timely mitotic exit or cytokinesis respectively. Interestingly, in S. pombe the cellular levels of Byr4p and Spg1p appear to be tightly coordinated, and proteasomal degradation of Byr4p is initiated if Byr4p and Spg1p are not complexed within the cell (Krapp et al., 2008). Although the stability of Bfa1 does 
not appear to be regulated across the cell cycle, the phosphorylation state of the protein changes dramatically with cell cycle stage (Hu et al., 2001). Specifically, phosphorylation by both Kin4 and Cdc5 are important to activate or inhibit the GAP activity of Bfa1 respectively (Hu et al., 2001; Geymonat et al., 2003; Maekawa et al., 2007). Thus in the budding yeast system, phosphorylation status, rather than proteolysis, regulates Bfa1-Bub2 GAP activity. This allows the GAP activity of Bfa1 to be regulated independently of its positive role in mediating Tem1 localization.

\section{Additional positive role for Bfa1 in cytokinesis regulation}

Our findings indicate that tem $1 \triangle C D C 15-U P$ cells have significant cytokinesis defects. These defects appear to be caused by the absence of TEM1, rather than the hyperactivation of $C D C 15$, since cells containing CDC15-UP alone do not exhibit chained cells (Figure 7B-D). Tem1 has a poorly understood role in cytokinesis that is independent of its role in promoting mitotic exit in anaphase. During cytokinesis, the septin ring splits, which allows for actomyosin ring (AMR) contraction, primary and secondary septum formation, and septum degradation in the daughter cell. This process leads to abscission between the mother and daughter cells (reviewed in Juanes and Piatti, 2016). Septin ring splitting occurs before AMR contraction during cytokinesis (Lippincott et al. 2001). It was previously found that in GAL-UPL-TEM1 net1-1 cells, where the mitotic exit requirement for Tem1 has been bypassed by mutation of the Cdc14 inhibitor CFI1/NET1, both septin ring splitting and AMR contraction are defective under TEM1-depletion conditions (Lippincott et al., 2001). Another cytokinesis activator Cyk1/lqg1 (IQGAP) is 
dispensable for septin ring splitting, but is required for activation of AMR contraction. Specifically, the GAP-related domain (GRD) of Cyk1/lqg1 is required for the protein to activate AMR contraction. Interestingly, Tem1 binds specifically to the GRD of the cytokinesis activator Cyk1/lqg and this raises the possibility that Tem1 regulates this protein's activity (Shannon and Li, 1999). How this regulation is achieved and where in the cell it occurs is not understood, since Cyk1/lqg1 localizes to the AMR at the budneck while Tem1 is not visible at this structure.

We found that the cytokinetic defects observed in tem $1 \triangle C D C 15-U P$ cells are suppressed by the overexpression of BFA1. Specifically, both the Dbf2 budneck localization defects, as well as the chained cell morphology defects, were ameliorated by GAL-BFA1 (Figure 7). Therefore, our work shows for the first time that BFA1 activates the process of cytokinesis independently of TEM1. Most likely, the positive role for Bfa1 in cytokinesis is through Mob1-Dbf2. Mob1-Dbf2 localization to the budneck is a requirement for actomyosin ring (AMR) contraction and for primary septum formation. Specifically, the F-BAR protein Hof1 is a direct target of Mob1-Dbf2 and thereby promotes AMR constriction (Frenz et al., 2000; Meitinger et al., 2010; Meitinger et al., 2011; Oh et al., 2012; Meitinger et al., 2013). In addition, we found that tem1 $C D C 15$ UP cells containing a Mob1-eGFP fusion do not exhibit defects in Mob1-eGFP localization to the budneck, nor do they display cytokinesis defects (data not shown). However, the MOB1-eGFP allele exhibits some hyperactivity, as evidenced by the localization of this protein to SPBs in metaphase (AS, unpublished observations). Therefore, we conclude that despite having normal Dbf2 kinase activation and cell cycle 
kinetics, tem1 $\triangle C D C 15-U P$ cells have defects in directing the active Mob1-Dbf2 complex to the budneck to fulfill its cytokinetic role (Rock and Amon, 2011; Figure 7). The accurate targeting of Mob1-Dbf2 during cytokinesis is enhanced by GAL-BFA1 (Figure 7A).

What could be the role of Bfa1 in promoting proper cytokinesis? In tem1A CDC15-UP cells, the polo kinase Cdc5 is required for mitotic exit (Rock and Amon, 2011). Recently it was shown that Bfa1 is required for the outer plaque dSPB localization of Cdc5 (Botchkarev et al., 2017). The role of Cdc5 localization to dSPBs is likely three-fold: 1) the phosphorylation and inhibition of Bfa1 GAP activity (Hu et al., 2001; Geymonat et al., 2003); 2) the phosphorylation and activation of the essential Cdc15 kinase (Rock and Amon, 2011); and 3) the initiation of septum formation during cytokinesis. To highlight the evidence in support of the role of Cdc5 dSPB localization in the activation of septum formation, previous studies showed that the $C D C 5 \triangle C-C D C 12$ allele produced a mutant fusion protein that localized to the budneck, but not to SPBs (Park et al., 2004). This mutant allele was capable of initiating mitotic exit in cells lacking BFA1. However, the Cdc5 $\Delta \mathrm{C}-\mathrm{Cdc} 12$ mutant protein was defective in initiating septum formation and cytokinesis, exhibiting chained cell growth. In contrast, the CDC5 $\triangle C$-CNM67 allele, which localized to SPBs but not to the budneck, exhibited proper mitotic exit, as well as efficient septum formation and cytokinesis. The authors concluded that Cdc5 SPB localization was required for efficient cytokinesis (Park et al., 2004). More recently, it was shown that Cdc5 inhibits the GTPase Cdc42 in late anaphase, which was also required for efficient septum formation (Atkins et al., 2013). However, it is not known 
whether the outer plaque SPB localization of Cdc5 is necessary for inhibition of Cdc42. In addition, it is not yet known whether the effects of Cdc5 on cytokinesis occur in a Mob1Dbf2 dependent manner. In this context, it would be important to examine whether GAL-BFA1 cells display either decreased or increased recruitment of Cdc5 to the dSPB in order to determine whether the effects of GAL-BFA1 on cytokinesis are mediated through Cdc5. While our results show that Dbf2 recruitment to the primary septum is reduced in the absence of TEM1, the links between Bfa1, Cdc5, and Mob1-Dbf2 function in cytokinesis have not been explored.

What is the significance of this dual positive and negative role for Bfa1 on Cdc14 regulation and mitotic exit? Why does $\mathrm{Bfa} 1$ have an additional positive function in cytokinesis regulation? One possible explanation is that the use of these same cellular components for multiple cell cycle functions allows for the temporal coupling of mitotic exit and cytokinesis (reviewed in Seshan and Amon, 2004). This is critical in order for cells to restrain cytokinesis until the completion of chromosome segregation. In the $S$. pombe system, the SIN pathway regulates septation and cytokinesis, but not mitotic exit (reviewed in Krapp and Simanis, 2008). Consequently, the deletion of Byr4p, the Bfa1 homolog in fission yeast, causes lethality due to resulting hyperactivity of Spg1p followed by multiple rounds of septation. Thus, in the absence of byr4, the process of septation is completely uncoordinated with other mitotic events (Song et al., 1996). Importantly, this is because cells lacking Byr4p are still capable of localizing Spg1p to SPBs, which allows for hyperactivation of the SIN (Sohrmann et al., 1998; Cerutti and Simanis, 1999). In the absence of BFA1 in S. cerevisiae, Tem1 localizes to both SPBs later 
in anaphase than in wild type cells, and cell cycle progression is normal in an

unperturbed cell cycle (Pereira et al., 2000; Valerio-Santiago and Monje-Casas, 2011).

Perhaps this is due to the fact that, as we have shown here, Bfa1 is a key anchor for

Tem1 at SPBs. The weaker localization of Tem1 in the absence of BFA1 may safeguard budding yeast cells from MEN hyperactivity and prevent bfa1 $\Delta$ lethality. Further investigation of the role of Bfa1 as a scaffold at the dSPB will lead to clarification of the role of this multi-functional protein in the efficient and accurate execution of late $\mathrm{M}$ phase events.

\section{MATERIALS AND METHODS}

\section{Yeast strains and growth conditions}

All yeast strains used in this study were derivatives of W303 (AS3). The CDC15-UP construct was described in Rock and Amon (2011). Culturing conditions were described in the figure legends.

\section{Fixed-cell imaging}

Indirect immunofluorescence was performed as described previously for $\alpha$-tubulin

(Tub1) and $\alpha$-HA to detect Cdc14-HA (Visintin et al., 1999). Images for Figures 1B, 2, 4A-

F, 5, and 6 were acquired using a Zeiss Axioplan 2 (Zeiss, Thornwood, NY) with a Hamamatsu Orca-R2 camera (Hamamatsu, Middlesex, NJ) and a 63x objective. Cells for 
Figures 3 and $4 G$ were fixed in a 4\% paraformaldehyde, $3.4 \%$ sucrose solution for 15

minutes. Cells were washed once in potassium phosphate/sorbitol buffer (1.2M sorbitol, 0.1M potassium phosphate, $\mathrm{pH} 7.5)$, and treated with $1 \%$ Triton X-100 for 5 minutes. Cells were washed again in potassium phosphate/sorbitol buffer and resuspended in potassium phosphate/sorbitol buffer containing 4', 6-diamidino-2-phenylindole dihydrochloride before imaging. Cells for Figure 7B-C were fixed in 3.7\% formaldehyde, 0.1M potassium phosphate, $\mathrm{pH} 6.4$ prior to sonication and imaging. These cells were imaged using a DeltaVision Elite microscope (GE Healthcare Bio-Sciences, Pittsburgh, PA) with an InsightSSI solid-state light source, a CoolSNAP HQ2 camera, and a 60x planApoN objective.

\section{Live-cell imaging}

Cells for Figure 7A were imaged directly from log phase cultures using a DeltaVision Elite microscope (GE Healthcare Bio-Sciences) with an InsightSSI solid-state light source, a CoolSNAP HQ2 camera, and a 60x plan-ApoN objective.

\section{Data availability}

All strains are available upon request. The authors affirm that all of the data necessary for confirming the conclusions made within this article are contained within the article and its figures.

\section{ACKNOWLEDGEMENTS}


We thank Angelika Amon and lan Campbell for providing a critical reading of the manuscript. We are grateful to Angelika Amon for generous sharing of strains and equipment. We also thank Chloe Egan, Aydah Mwangi and Micah Tilove for technical support. 


\section{REFERENCES}

1. Alexandru G, Zachariae W, Schleiffer A, Nasmyth K. Sister chromatid separation and chromosome re-duplication are regulated by different mechanisms in response to spindle damage. EMBO J. 1999 May 17;18(10):2707-21.

2. Asakawa K, Yoshida S, Otake F, Toh-e A. A novel functional domain of Cdc15 kinase is required for its interaction with Tem1 GTPase in Saccharomyces cerevisiae. Genetics. 2001 April 01;157(4):1437-50.

3. Atkins BD, Yoshida S, Saito K, Wu CF, Lew DJ, Pellman D. Inhibition of Cdc42 during mitotic exit is required for cytokinesis. J Cell Biol. 2013 July 22;202(2):231-40.

4. Bardin AJ, Visintin R, Amon A. A mechanism for coupling exit from mitosis to partitioning of the nucleus. Cell. 2000 July 07;102(1):21-31.

5. Botchkarev VV, Garabedian MV, Lemos B, Paulissen E, Haber JE. The budding yeast Polo-like kinase localizes to distinct populations at centrosomes during mitosis. Mol Biol Cell. 2017 April 15;28(8):1011-20.

6. Caydasi AK, Ibrahim B, Pereira G. Monitoring spindle orientation: Spindle position checkpoint in charge. Cell Div. 2010 December 11;5:28.

7. Cenamor R, Jimenez J, Cid VJ, Nombela C, Sanchez M. The budding yeast Cdc15 localizes to the spindle pole body in a cell-cycle-dependent manner. Mol Cell Biol Res Commun. 1999 December 01;2(3):178-84.

8. Cerutti L, Simanis V. Asymmetry of the spindle pole bodies and spg1p GAP segregation during mitosis in fission yeast. J Cell Sci. 1999 July 01;112 ( Pt 14)(Pt 14):2313-21.

9. Chan LY, Amon A. The protein phosphatase 2A functions in the spindle position checkpoint by regulating the checkpoint kinase Kin4. Genes Dev. 2009 July 15;23(14):1639-49.

10. Fesquet D, Fitzpatrick PJ, Johnson AL, Kramer KM, Toyn JH, Johnston LH. A Bub2pdependent spindle checkpoint pathway regulates the Dbf2p kinase in budding yeast. EMBO J. 1999 May 04;18(9):2424-34.

11. Fraschini R, D'Ambrosio C, Venturetti M, Lucchini G, Piatti S. Disappearance of the budding yeast Bub2-Bfa1 complex from the mother-bound spindle pole contributes to mitotic exit. J Cell Biol. 2006 January 30;172(3):335-46.

12. Fraschini R, Formenti E, Lucchini G, Piatti S. Budding yeast Bub2 is localized at spindle pole bodies and activates the mitotic checkpoint via a different pathway from Mad2. J Cell Biol. 1999 May 31;145(5):979-91. 
13. Frenz LM, Lee SE, Fesquet $D$, Johnston $L H$. The budding yeast Dbf2 protein kinase localises to the centrosome and moves to the bud neck in late mitosis. J Cell Sci. 2000 October 01;113 Pt 19:3399-408.

14. Furge KA, Wong K, Armstrong J, Balasubramanian M, Albright CF. Byr4 and Cdc16 form a two-component GTPase-activating protein for the Spg1 GTPase that controls septation in fission yeast. Curr Biol. 1998 August 27;8(17):947-54.

15. Geymonat M, Jensen S, Johnston LH. Mitotic exit: the Cdc14 double cross. Curr Biol. 2002 July 23;12(14):482.

16. Geymonat M, Spanos A, de Bettignies G, Sedgwick SG. Lte1 contributes to Bfa1 localization rather than stimulating nucleotide exchange by Tem1. J Cell Biol. 2009 November 16;187(4):497-511.

17. Geymonat M, Spanos A, Walker PA, Johnston LH, Sedgwick SG. In vitro regulation of budding yeast Bfa1/Bub2 GAP activity by Cdc5. J Biol Chem. 2003 April 25;278(17):14591-4.

18. Gruneberg U, Campbell K, Simpson C, Grindlay J, Schiebel E. Nud1p links astral microtubule organization and the control of exit from mitosis. EMBO J. 2000 December 01;19(23):6475-88.

19. Gryaznova Y, Koca Caydasi A, Malengo G, Sourjik V, Pereira G. A FRET-based study reveals site-specific regulation of spindle position checkpoint proteins at yeast centrosomes. Elife. 2016 May 09;5:10.7554/eLife.14029.

20. Hu F, Wang Y, Liu D, Li Y, Qin J, Elledge SJ. Regulation of the Bub2/Bfa1 GAP complex by Cdc5 and cell cycle checkpoints. Cell. 2001 November 30;107(5):655-65.

21. Jaspersen SL, Charles JF, Morgan DO. Inhibitory phosphorylation of the APC regulator Hct1 is controlled by the kinase $\mathrm{Cdc} 28$ and the phosphatase Cdc14. Curr Biol. 1999 March 11;9(5):227-36.

22. Juanes MA, Piatti S. The final cut: cell polarity meets cytokinesis at the bud neck in S. cerevisiae. Cell Mol Life Sci. 2016 August 01;73(16):3115-36.

23. Kim J, Jang SS, Song K. Different levels of Bfa1/Bub2 GAP activity are required to prevent mitotic exit of budding yeast depending on the type of perturbations. Mol Biol Cell. 2008 October 01;19(10):4328-40.

24. Kim J, Luo G, Bahk YY, Song K. Cdc5-dependent asymmetric localization of bfa1 finetunes timely mitotic exit. PLoS Genet. 2012 January 01;8(1):e1002450.

25. Komarnitsky SI, Chiang YC, Luca FC, Chen J, Toyn JH, Winey M, et al. DBF2 protein kinase binds to and acts through the cell cycle-regulated MOB1 protein. Mol Cell Biol. 1998 April 01;18(4):2100-7. 
26. Krapp A, Collin P, Cano Del Rosario E, Simanis V. Homoeostasis between the GTPase Spg1p and its GAP in the regulation of cytokinesis in S. pombe. J Cell Sci. 2008 March 01;121(Pt 5):601-8.

27. Krapp A, Simanis V. An overview of the fission yeast septation initiation network (SIN). Biochem Soc Trans. 2008 June 01;36(Pt 3):411-5.

28. Lee SE, Frenz LM, Wells NJ, Johnson AL, Johnston LH. Order of function of the budding-yeast mitotic exit-network proteins Tem1, Cdc15, Mob1, Dbf2, and Cdc5. Curr Biol. 2001 May 15;11(10):784-8.

29. Li C, Furge KA, Cheng QC, Albright CF. Byr4 localizes to spindle-pole bodies in a cell cycle-regulated manner to control $\mathrm{Cdc} 7$ localization and septation in fission yeast. J Biol Chem. 2000 May 12;275(19):14381-7.

30. Li R. Bifurcation of the mitotic checkpoint pathway in budding yeast. Proc Natl Acad Sci U S A. 1999 April 27;96(9):4989-94.

31. Luca FC, Winey M. MOB1, an essential yeast gene required for completion of mitosis and maintenance of ploidy. Mol Biol Cell. 1998 January 01;9(1):29-46.

32. Maekawa H, Priest C, Lechner J, Pereira G, Schiebel E. The yeast centrosome translates the positional information of the anaphase spindle into a cell cycle signal. J Cell Biol. 2007 November 05;179(3):423-36.

33. Mah AS, Jang J, Deshaies RJ. Protein kinase Cdc15 activates the Dbf2-Mob1 kinase complex. Proc Natl Acad Sci U S A. 2001 June 19;98(13):7325-30.

34. Meitinger F, Boehm ME, Hofmann A, Hub B, Zentgraf $H$, Lehmann WD, et al. Phosphorylation-dependent regulation of the F-BAR protein Hof1 during cytokinesis. Genes Dev. 2011 April 15;25(8):875-88.

35. Meitinger F, Palani S, Hub B, Pereira G. Dual function of the NDR-kinase Dbf2 in the regulation of the F-BAR protein Hof1 during cytokinesis. Mol Biol Cell. 2013 May 01;24(9):1290-304.

36. Meitinger F, Petrova B, Lombardi IM, Bertazzi DT, Hub B, Zentgraf H, et al. Targeted localization of Inn1, Cyk3 and Chs2 by the mitotic-exit network regulates cytokinesis in budding yeast. J Cell Sci. 2010 June 01;123(Pt 11):1851-61.

37. Molk JN, Schuyler SC, Liu JY, Evans JG, Salmon ED, Pellman D, et al. The differential roles of budding yeast Tem1 1 , Cdc15p, and Bub2p protein dynamics in mitotic exit. Mol Biol Cell. 2004 April 01;15(4):1519-32.

38. Musacchio A, Salmon ED. The spindle-assembly checkpoint in space and time. Nat Rev Mol Cell Biol. 2007 May 01;8(5):379-93. 
39. Oh Y, Chang KJ, Orlean P, Wloka C, Deshaies R, Bi E. Mitotic exit kinase Dbf2 directly phosphorylates chitin synthase Chs 2 to regulate cytokinesis in budding yeast. Mol Biol Cell. 2012 July 01;23(13):2445-56.

40. Park JE, Park CJ, Sakchaisri K, Karpova T, Asano S, McNally J, et al. Novel functional dissection of the localization-specific roles of budding yeast polo kinase Cdc5p. Mol Cell Biol. 2004 November 01;24(22):9873-86.

41. Pereira G, Hofken T, Grindlay J, Manson C, Schiebel E. The Bub2p spindle checkpoint links nuclear migration with mitotic exit. Mol Cell. 2000 July 01;6(1):1-10.

42. Pereira G, Schiebel E. The role of the yeast spindle pole body and the mammalian centrosome in regulating late mitotic events. Curr Opin Cell Biol. 2001 December $01 ; 13(6): 762-9$.

43. Ro HS, Song S, Lee KS. Bfa1 can regulate Tem1 function independently of Bub2 in the mitotic exit network of Saccharomyces cerevisiae. Proc Natl Acad Sci U S A. 2002 April 16;99(8):5436-41.

44. Rock JM, Amon A. Cdc15 integrates Tem1 GTPase-mediated spatial signals with Polo kinase-mediated temporal cues to activate mitotic exit. Genes Dev. 2011 September $15 ; 25(18): 1943-54$.

45. Rock JM, Amon A. The FEAR network. Curr Biol. 2009 December 15;19(23):1063.

46. Rock JM, Lim D, Stach L, Ogrodowicz RW, Keck JM, Jones MH, et al. Activation of the yeast Hippo pathway by phosphorylation-dependent assembly of signaling complexes. Science. 2013 May 17;340(6134):871-5.

47. Scarfone I, Piatti S. Coupling spindle position with mitotic exit in budding yeast: The multifaceted role of the small GTPase Tem1. Small GTPases. 2015 October 02;6(4):196201.

48. Scarfone I, Venturetti M, Hotz M, Lengefeld J, Barral Y, Piatti S. Asymmetry of the budding yeast Tem 1 GTPase at spindle poles is required for spindle positioning but not for mitotic exit. PLoS Genet. 2015 February 06;11(2):e1004938.

49. Seshan A, Amon A. Linked for life: temporal and spatial coordination of late mitotic events. Curr Opin Cell Biol. 2004 February 01;16(1):41-8.

50. Shannon KB, Li R. The multiple roles of Cyk1p in the assembly and function of the actomyosin ring in budding yeast. Mol Biol Cell. 1999 February 01;10(2):283-96.

51. Shou W, Seol JH, Shevchenko A, Baskerville C, Moazed D, Chen ZW, et al. Exit from mitosis is triggered by Tem1-dependent release of the protein phosphatase Cdc14 from nucleolar RENT complex. Cell. 1999 April 16;97(2):233-44. 
52. Sohrmann M, Schmidt S, Hagan I, Simanis V. Asymmetric segregation on spindle poles of the Schizosaccharomyces pombe septum-inducing protein kinase Cdc7p. Genes Dev. 1998 January 01;12(1):84-94.

53. Song K, Mach KE, Chen CY, Reynolds T, Albright CF. A novel suppressor of ras1 in fission yeast, byr4, is a dosage-dependent inhibitor of cytokinesis. J Cell Biol. 1996 June 01;133(6):1307-19.

54. Stegmeier F, Amon A. Closing mitosis: the functions of the Cdc14 phosphatase and its regulation. Annu Rev Genet. 2004;38:203-32.

55. Valerio-Santiago M, Monje-Casas F. Tem1 localization to the spindle pole bodies is essential for mitotic exit and impairs spindle checkpoint function. J Cell Biol. 2011 February 21;192(4):599-614.

56. Visintin R, Amon A. Regulation of the mitotic exit protein kinases Cdc15 and Dbf2. Mol Biol Cell. 2001 October 01;12(10):2961-74.

57. Visintin R, Hwang ES, Amon A. Cfi1 prevents premature exit from mitosis by anchoring Cdc14 phosphatase in the nucleolus. Nature. 1999 April 29;398(6730):818-23.

58. Xu S, Huang HK, Kaiser P, Latterich M, Hunter T. Phosphorylation and spindle pole body localization of the $\mathrm{Cdc} 15 \mathrm{p}$ mitotic regulatory protein kinase in budding yeast. Curr Biol. 2000 March 23;10(6):329-32.

\section{FIGURE LEGENDS}

Figure 1 BFA1 has a BUB2-independent late anaphase function. A) Ten-fold serial dilutions of wild type (AS3), GAL-GFP-BFA1 (AS5), and GAL-GFP-BFA1 bub2 $\triangle$ (AS206) cells were spotted onto YEPD or YEP+Raffinose+Galactose (YEPRG) plates and incubated at $30^{\circ} \mathrm{C}$ for two days before imaging. B) Galactose was added at time 0 to the log phase, asynchronous, YEP+Raffinose (YEPR) cultures of the indicated genotypes grown at $21^{\circ} \mathrm{C}$ in order to induce the overexpression of GAL-GFP-BFA1. Samples were taken at the indicated times and processed for tubulin immunofluorescence. The percentage of anaphase cells was determined at each time point ( $n=100-200$ cells). 
Figure 2 Cdc14 activation is impaired in GAL-BFA1 cells. A) Log phase cultures of wild type (AS138) and GAL-BFA1 (AS24) cells carrying a CDC14-3HA fusion growing at $21^{\circ} \mathrm{C}$ in YEPR were arrested in $\mathrm{G} 1$ using alpha-factor pheromone ( $5 \mu \mathrm{g} / \mathrm{mL})$. At 1.5 hours into the arrest, galactose (GAL) was added to induce expression of GAL-BFA1. Cells were released from the G1 arrest after 3 hours into YEPRG. Cells were collected at the times indicated to process samples for tubulin and Cdc14-HA immunofluorescence. The percentage of cells with anaphase spindle morphology was quantified for each time point and is indicated in the chart below the graph. The percentage of cells with Cdc14 localized to the nucleus and cytoplasm (full Cdc14 release) was quantified in wild type (circles) and GAL-BFA1 (squares) cells ( $N=100-200$ cells). B) Log phase wild type (AS3), GAL-BFA1 (AS5), cfi1 (AS121), and cfi1 GAL-BFA1 (AS141) were grown in YEPRG at $21^{\circ} \mathrm{C}$ for three hours to induce the overexpression of BFA1 and samples were collected and processed for tubulin immunofluorescence. The percentage of cells with anaphase spindle morphology was quantified ( $N=200$ cells).

Figure 3 Tem1 localization to SPBs is perturbed in GAL-BFA1 cells. A) A GAL-GFP-BFA1 strain (AS5) was grown to log phase in YEPR at $21^{\circ} \mathrm{C}$ and the cells were arrested in $\mathrm{G} 1$ with alpha-factor pheromone $(5 \mu \mathrm{g} / \mathrm{mL})$. Cells were released from the arrest in YEPRG and the overexpression of BFA1 was induced for a total of 4.5 hours. Cells were fixed with paraformaldehyde prior to imaging. Representative anaphase cells are shown. The percentage of anaphase cells with Bfa1 localized to the dSPB, both the dSPB and the 
mSPB (both), or to neither SPB was quantified $(n=133)$. Asynchronous no tag wild type (AS3) cells were also imaged. B and C) Wild type (AS15) and GAL-BFA1 strains (AS79) containing the Tem1-GFP fusion were grown in YEPR (- Gal) or YEPRG (+ Gal) media for three hours at $21^{\circ} \mathrm{C}$ before paraformaldehyde fixation and imaging. Anaphase cells were identified by DAPI morphology and 50 to 100 cells were analyzed in each condition. Representative anaphase cells are shown in B). Tem1-GFP is displayed in green and the DNA is shown in blue. Tem1-GFP localization to SPBs is indicated by the white arrowheads. C) The percentage of anaphase cells in each sample with Tem1-GFP localized to one or both SPBs (black bars) or delocalized throughout the cell (hatched bars) was plotted.

Figure 4 Restoration of Tem1 localization is not sufficient to suppress the effects of GAL-BFA1 Log phase YEPR cultures of wild type (AS138), GAL-BFA1 (AS24), TEM1-eGFPCNM67 (AS109), and GAL-BFA1 TEM1-eGFP-CNM67 (AS188) cells carrying a CDC14-3HA fusion were arrested with alpha-factor pheromone $(5 \mu \mathrm{g} / \mathrm{mL})$ at $21^{\circ} \mathrm{C}$. At 1.5 hours into the arrest, galactose was added. The cells were released from the arrest after 3 hours into YEPRG media. Cells were collected at the times indicated to process for tubulin and CDC14-HA immunofluorescence. A-D) The percentage of cells with metaphase (squares) and anaphase (triangles) spindle morphology was quantified for each time point $(\mathrm{N}=$ 100-200 cells). E) The percentage of anaphase cells at each time point from (A) - (D) was plotted for comparison. F) The percentage of anaphase cells of the genotypes indicated with Cdc14 sequestered (black), partially released (dotted) or fully released (white) was 
plotted ( $\mathrm{N}=100$ cells). G) TEM1-eGFP-CNM67 (AS19) and GAL-BFA1 TEM1-eGFP-CNM67 (AS36) cells were grown at $21^{\circ} \mathrm{C}$ in YEPRG for three hours and cells were imaged after paraformaldehyde fixation. Representative GAL-BFA1 TEM1-eGFP-CNM67 cells are shown. H) The percentage of TEM1-eGFP-CNM67 (black bars) or GAL-BFA1 TEM1-eGFPCNM67 (stippled bars) anaphase cells with Tem1-eGFP-CNM67 localized equally on both SPBs, brighter on the dSPB, brighter on the mSPB, or located only on the dSPB was quantified ( $N=100$ cells).

Figure 5 The TEM1- $2 \mu$ allele does not suppress GAL-BFA1. A - D) Log phase cultures of wild type + YEP $2 \mu$ (empty vector; AS207), wild type + TEM1-2 $\mu$ (AS209), GAL-BFA1 + YEP $2 \mu$ (AS211), and GAL-BFA1 + TEM1- $2 \mu$ (AS213) were arrested using alpha-factor pheromone $(5 \mu \mathrm{g} / \mathrm{mL})$ at $21^{\circ} \mathrm{C}$ in SC - Leu + Raffinose media. At 1.5 hours into the arrest, galactose was added to the cells to induce the expression of GAL-BFA1. Cells were released from the arrest into SC -Leu + Raffinose +Galactose media after 3 hours. Samples of cells were collected at the indicated times and processed for tubulin immunofluorescence. The percentages of metaphase and anaphase cells were determined at each time point $(\mathrm{N}=100-200)$. E) The percentage of anaphase cells at each time point from $(A)-(D)$ was plotted for comparison.

Figure 6 The GAL-TEM1 allele displays robust suppression of GAL-BFA1. A - D) Log phase wild type (AS3), GAL-BFA1 (AS413), GAL-TEM1 (AS401), and GAL-BFA1 GAL-TEM1 
(AS414) cells were arrested in YEPR media at $25^{\circ} \mathrm{C}$ using alpha-factor pheromone $(5$ $\mu \mathrm{g} / \mathrm{mL}$ ). At 1.5 hours into the arrest, galactose was added to the cells to induce the expression of GAL-BFA1 and/or GAL-TEM1. Cells were released from the arrest into YEPRG media after 3 hours. Samples of cells were collected the indicated times and were processed for tubulin immunofluorescence. The percentages of metaphase and anaphase cells were determined at each time point $(N=100-200)$. E) The percentage of anaphase cells at each time point from (A) - (D) was plotted for comparison.

Figure 7 Dbf2 budneck localization and cytokinesis defects in tem $1 \Delta$ cells are

\section{ameliorated by GAL-BFA1.}

Log phase samples of wild type (AS358), tem1 $\triangle$ CDC15-UP (AS431), and tem1 $\triangle C D C 15$ UP GAL-BFA1 (AS394) strains harboring Dbf2-eGFP and Spc42-mCherry fusions grown at $23^{\circ} \mathrm{C}$ in SC+RG media were collected and imaged. A) Large budded cells with Spc42 distributed in mother and daughter cells were identified and the percentage of cells with Dbf2-eGFP localized only to one or more spindle pole body (SPB only), localized only to the budneck (Budneck only), to SPBs and to the budneck (Budneck + SPB), or delocalized (None) was quantified in each strain ( $N=120$ cells). B) Wild type (AS3); CDC15UP (AS118); CDC15-UP GAL-GFP-BFA1 (AS119); tem1 $\triangle$ CDC15-UP (AS259); and tem1 $\Delta$ CDC15-UP GAL-GFP-BFA1 (AS493) cells were grown to log phase in SC+RG media at $23^{\circ} \mathrm{C}$, fixed in formaldehyde, and subjected to brief sonication. The percentage of cells with one cell body, two connected cell bodies, or three connected cell bodies was 
bioRxiv preprint doi: https://doi.org/10.1101/352427; this version posted June 20,2018 . The copyright holder for this preprint (which was not certified by peer review) is the author/funder. All rights reserved. No reuse allowed without permission.

quantified $(\mathrm{n}=200)$. Representative DIC microscopic images CDC15-UP (C); tem1 $\Delta$

CDC15-UP (D); and tem1 $\triangle$ CDC15-UP GAL-GFP-BFA1 (E) cells are shown. 
Whalen_Figure 1

A

YEPD

YEPRG
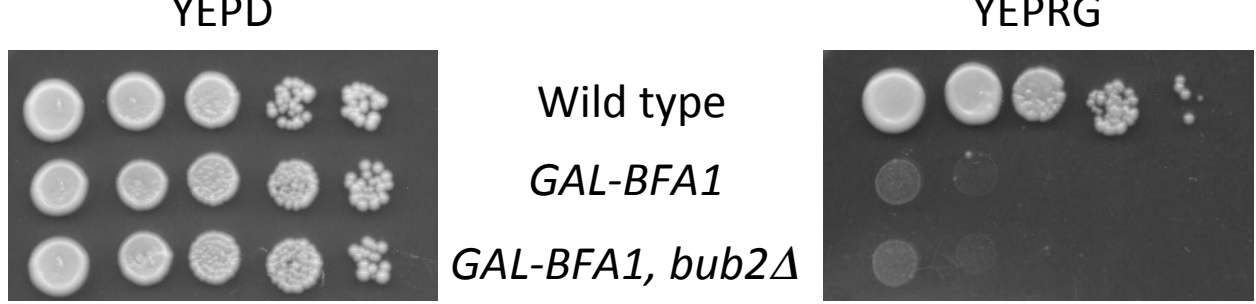

B

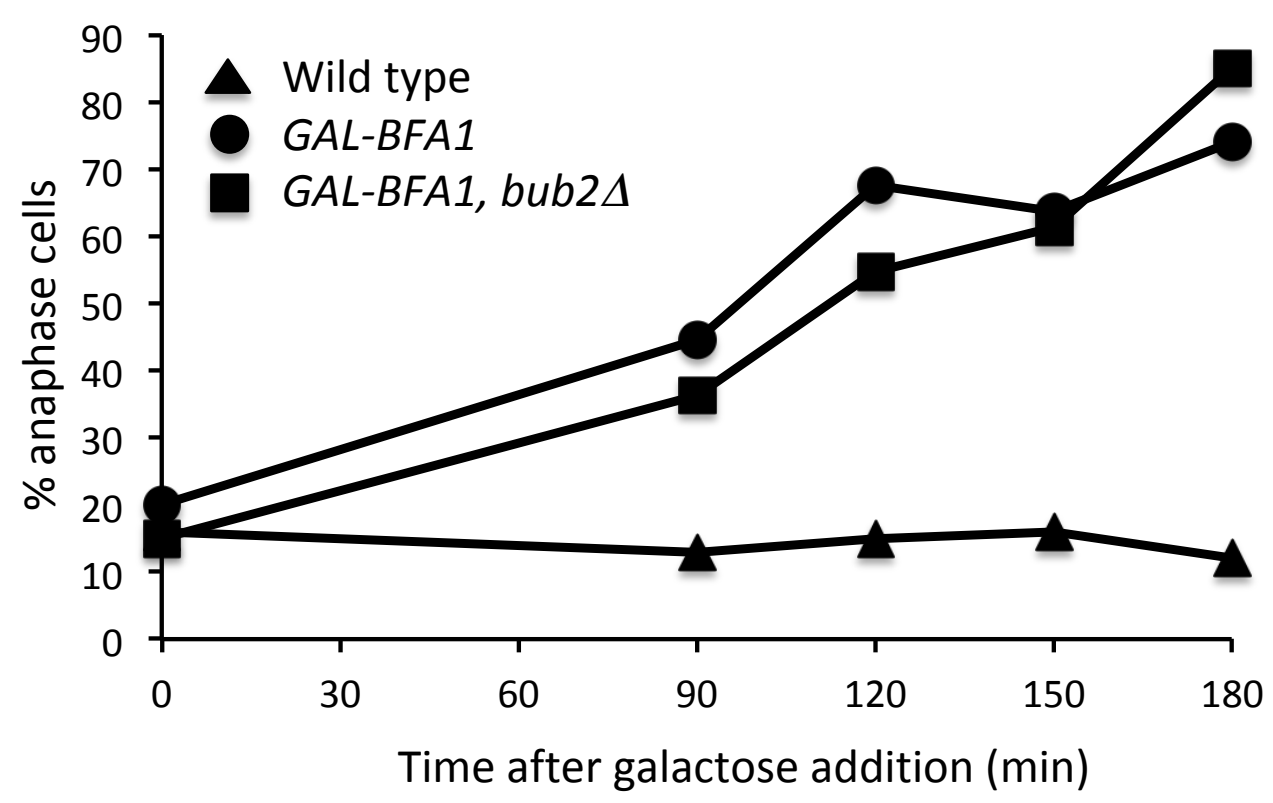


Whalen_Figure 2
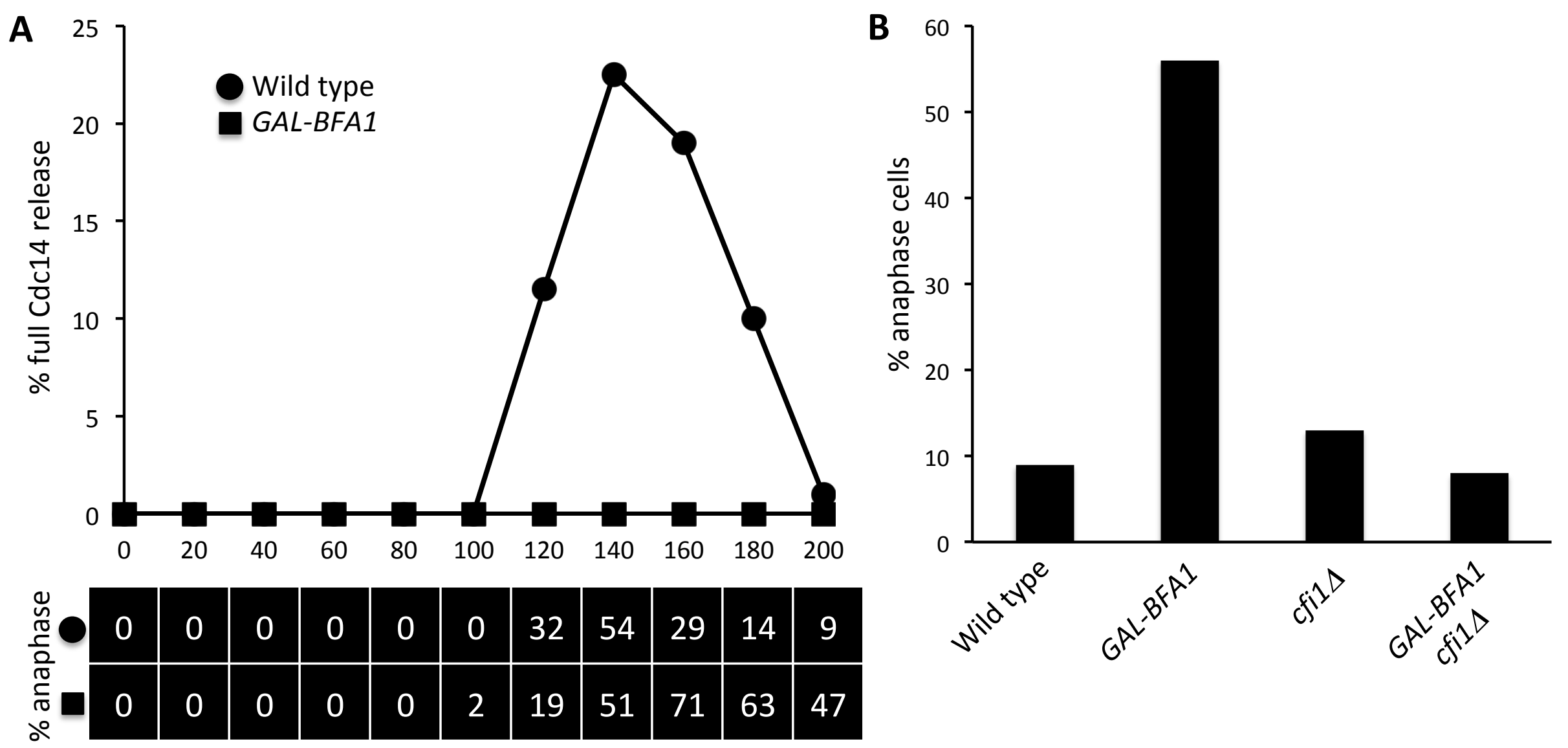
Whalen_Figure 3

A
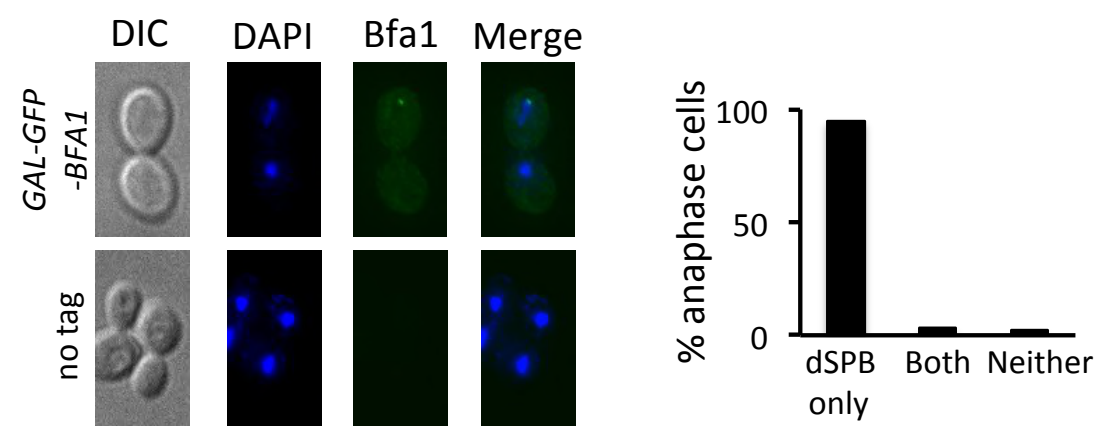

B
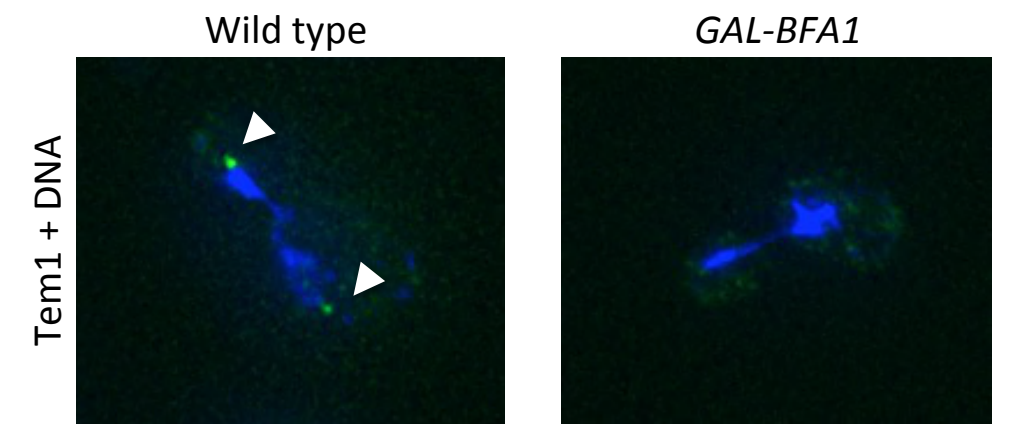

C

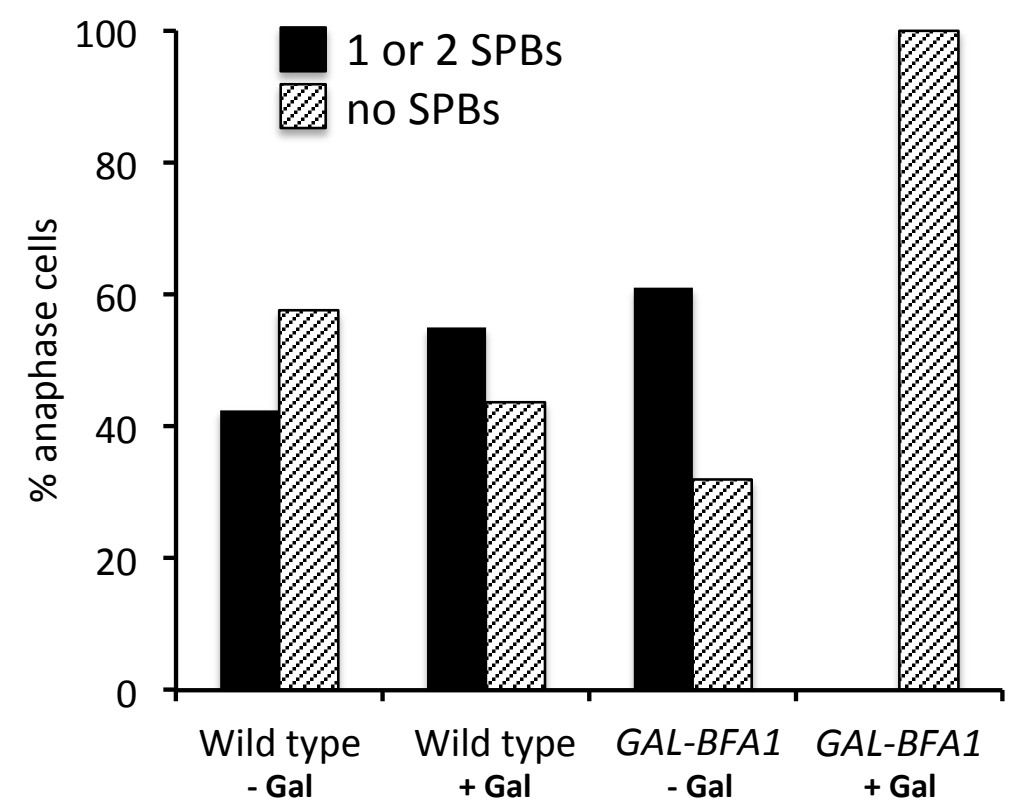



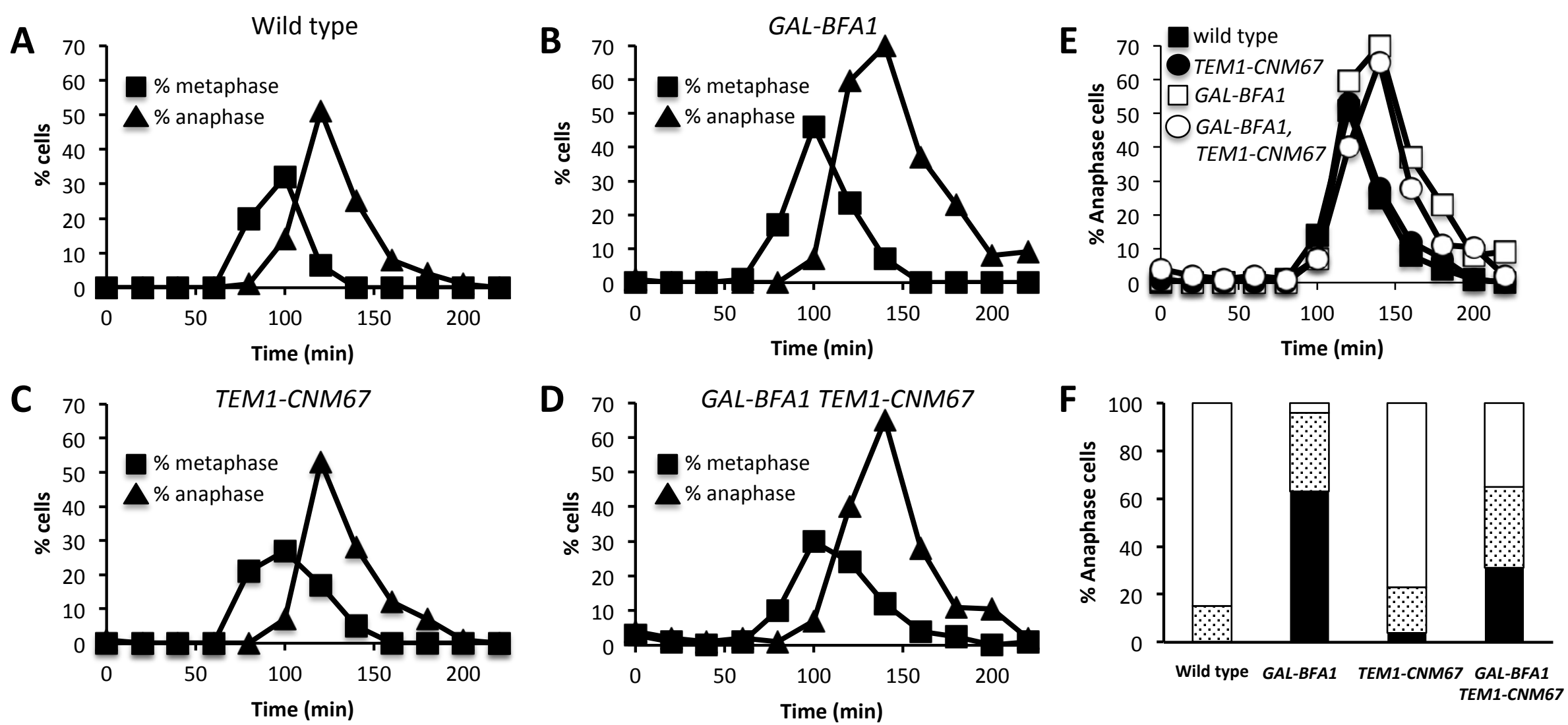

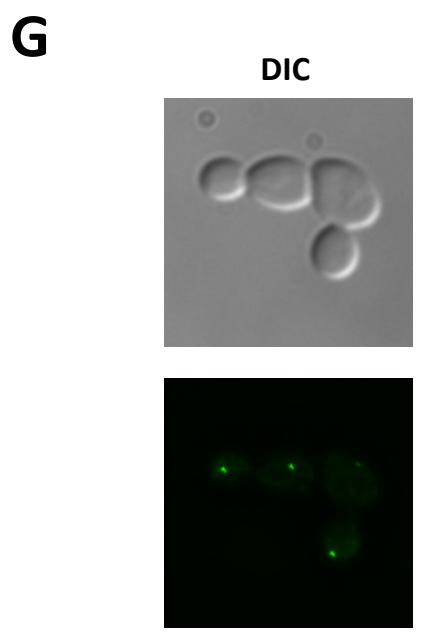

Tem1-eGFP-CNM67

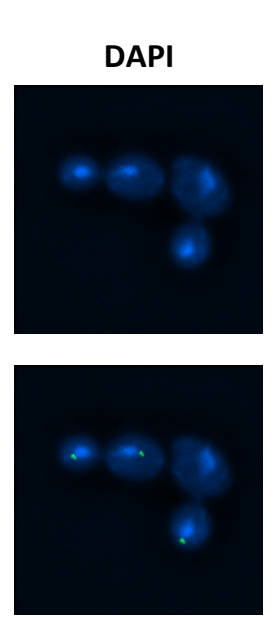

Merge
H
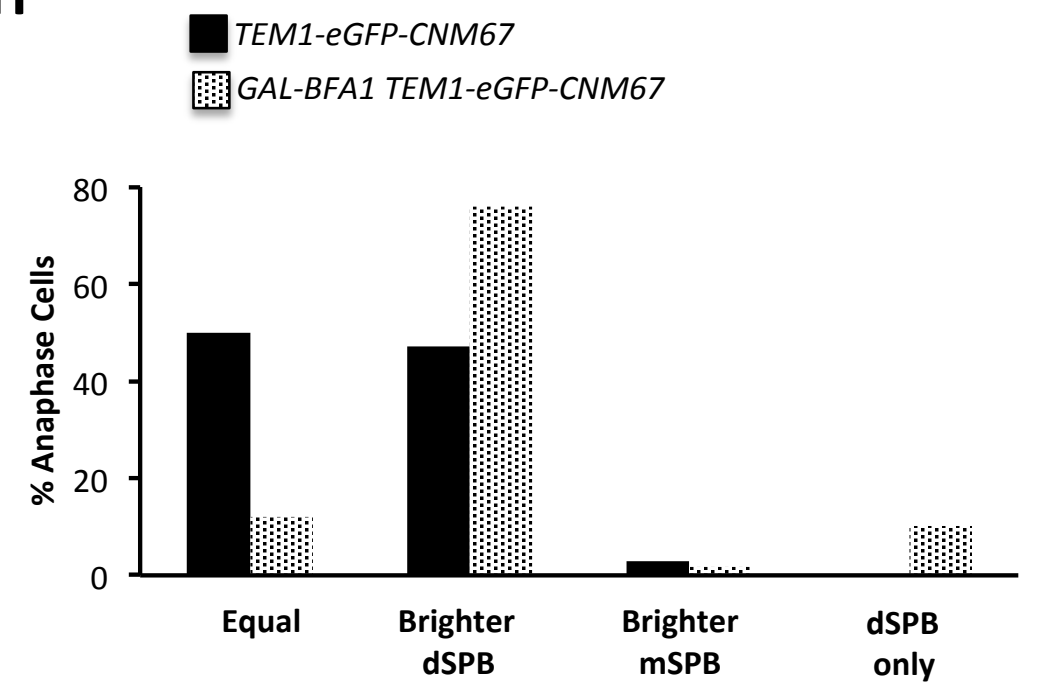

Whalen_Figure 4 

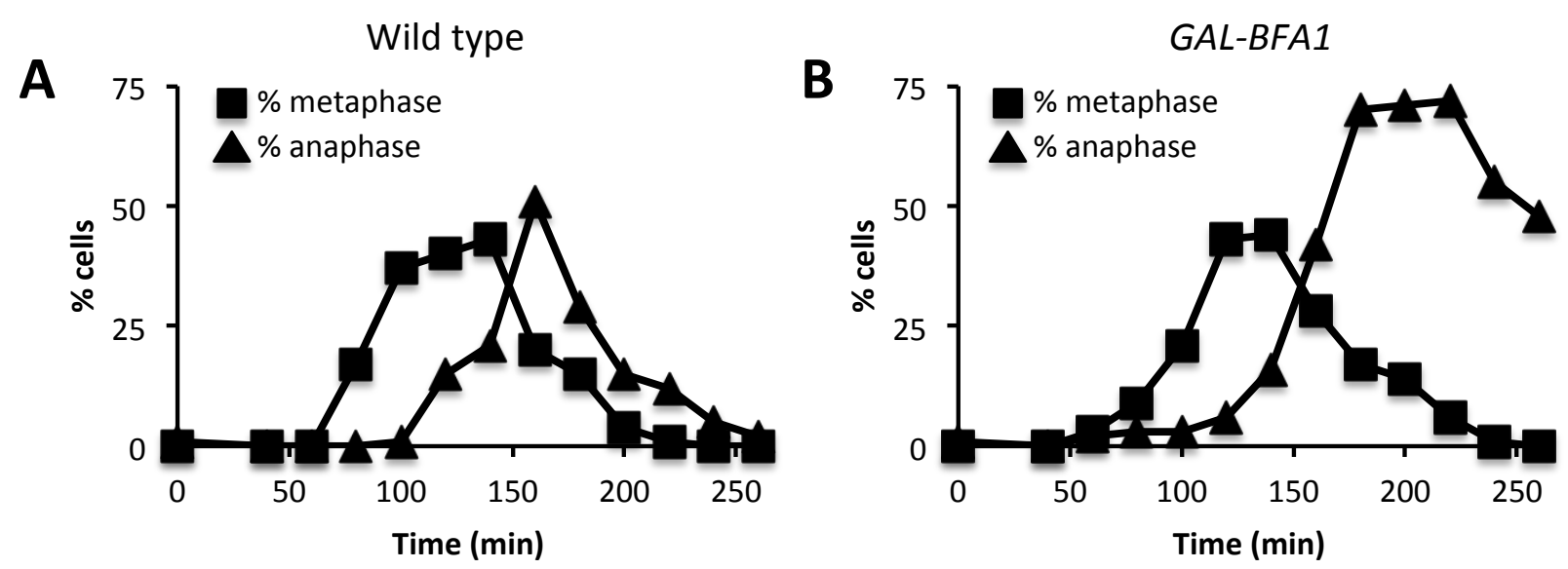

Whalen_Figure 5
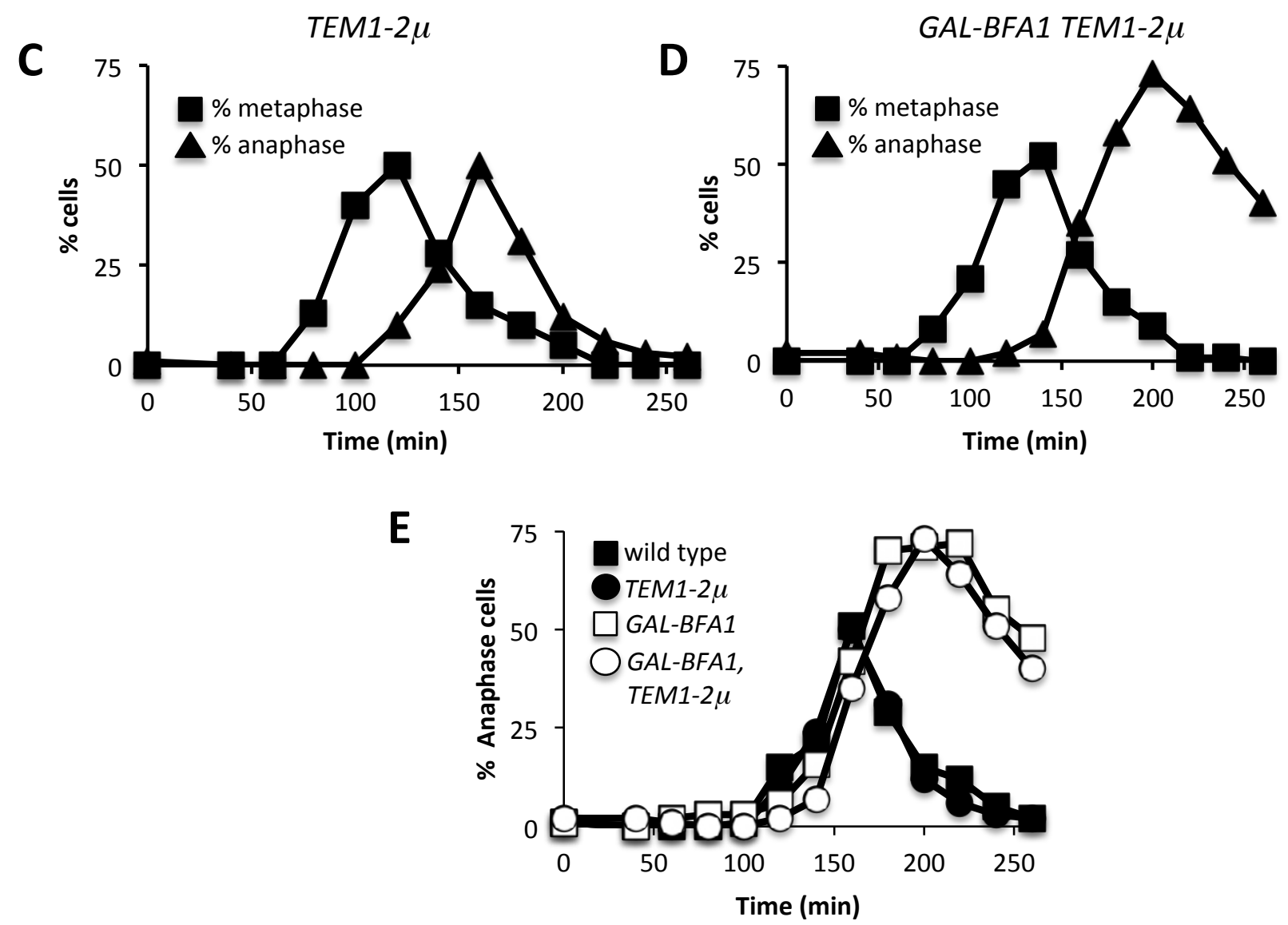
Whalen_Figure 6

Wild type

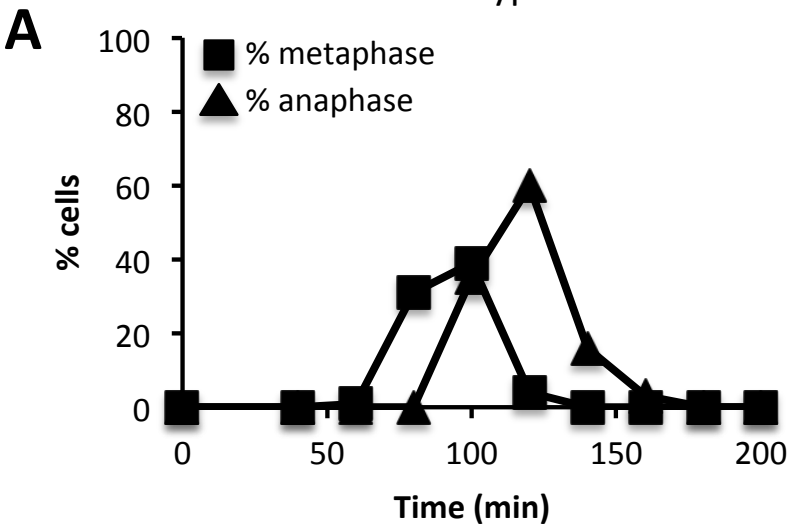

GAL-TEM1

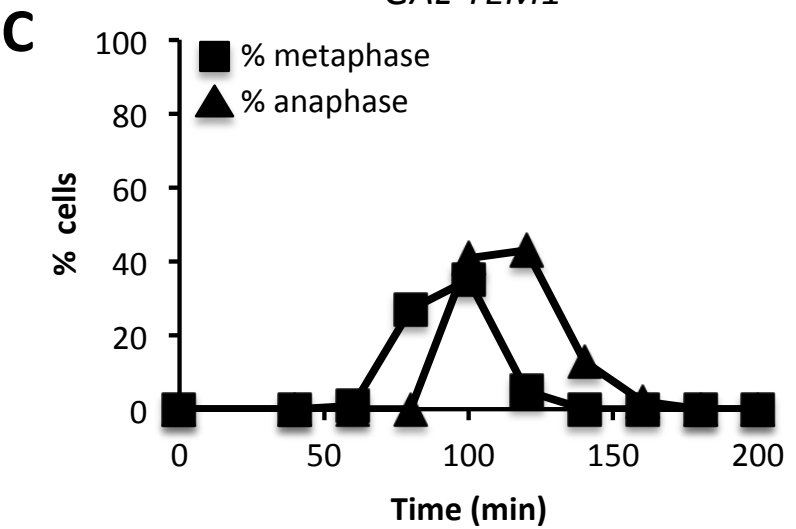

GAL-BFA1
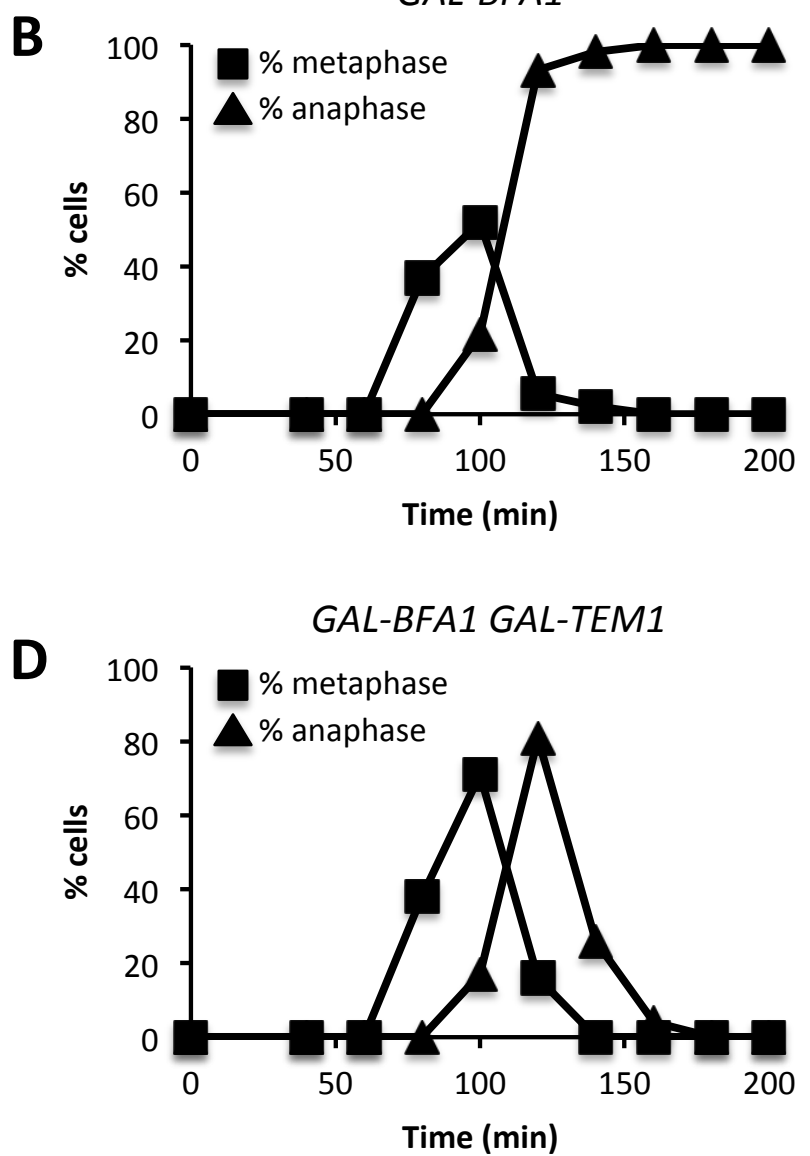

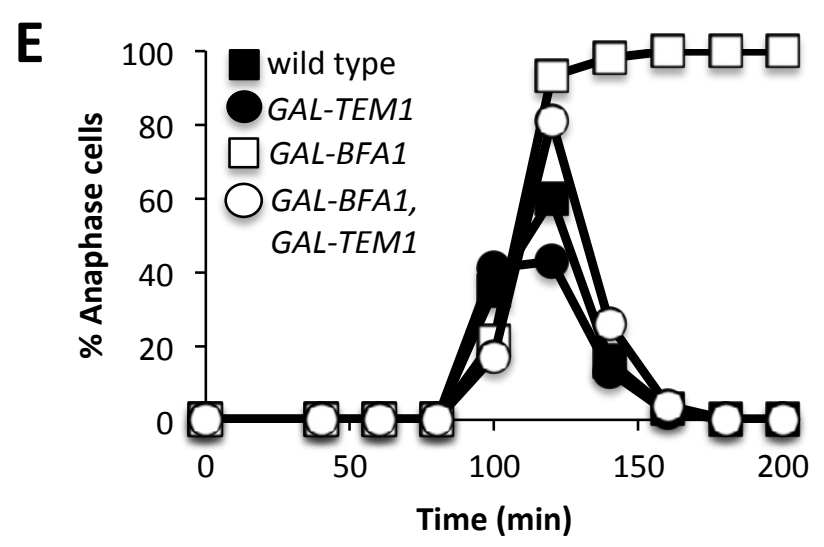




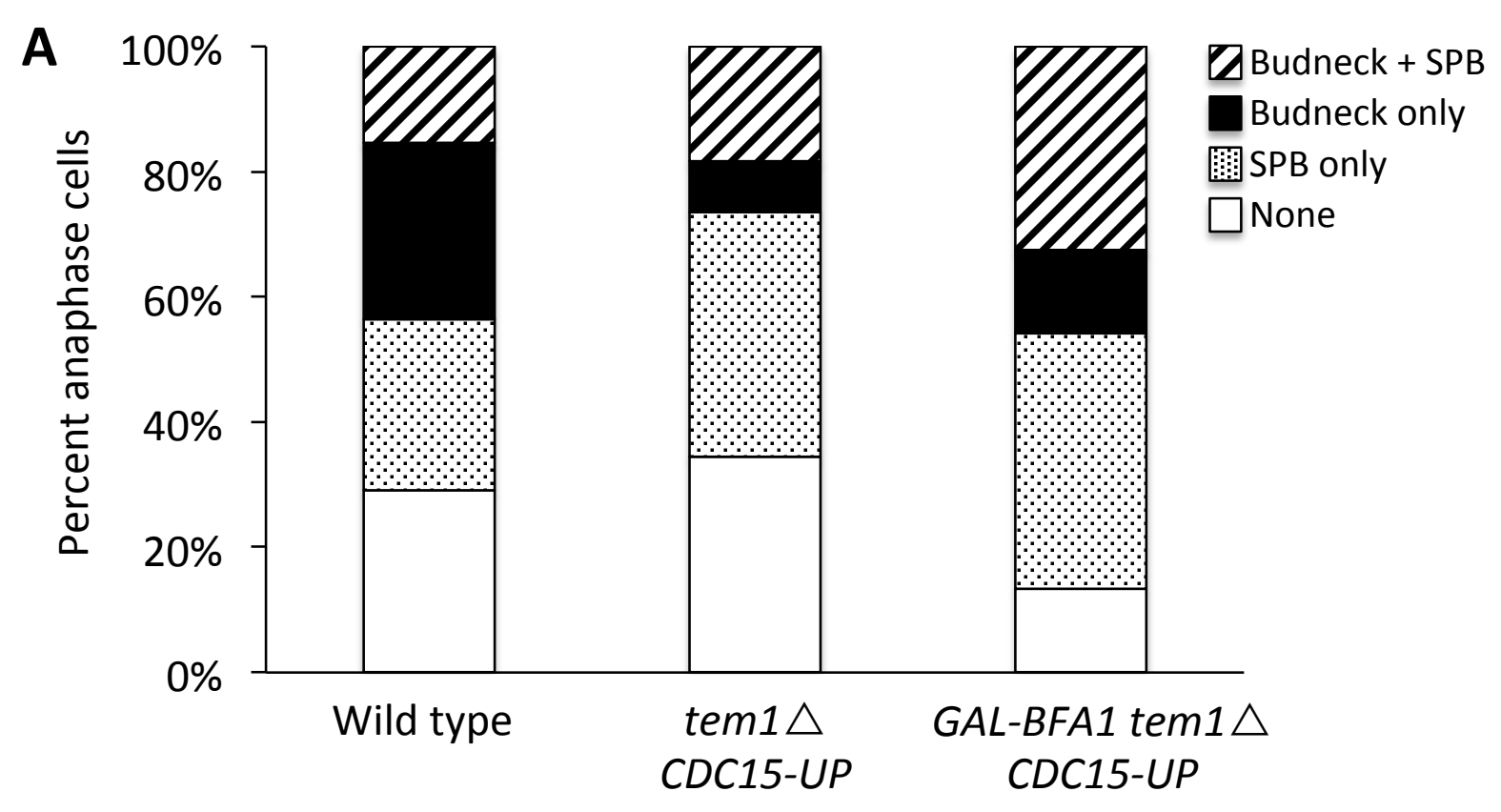

Whalen_Figure 7

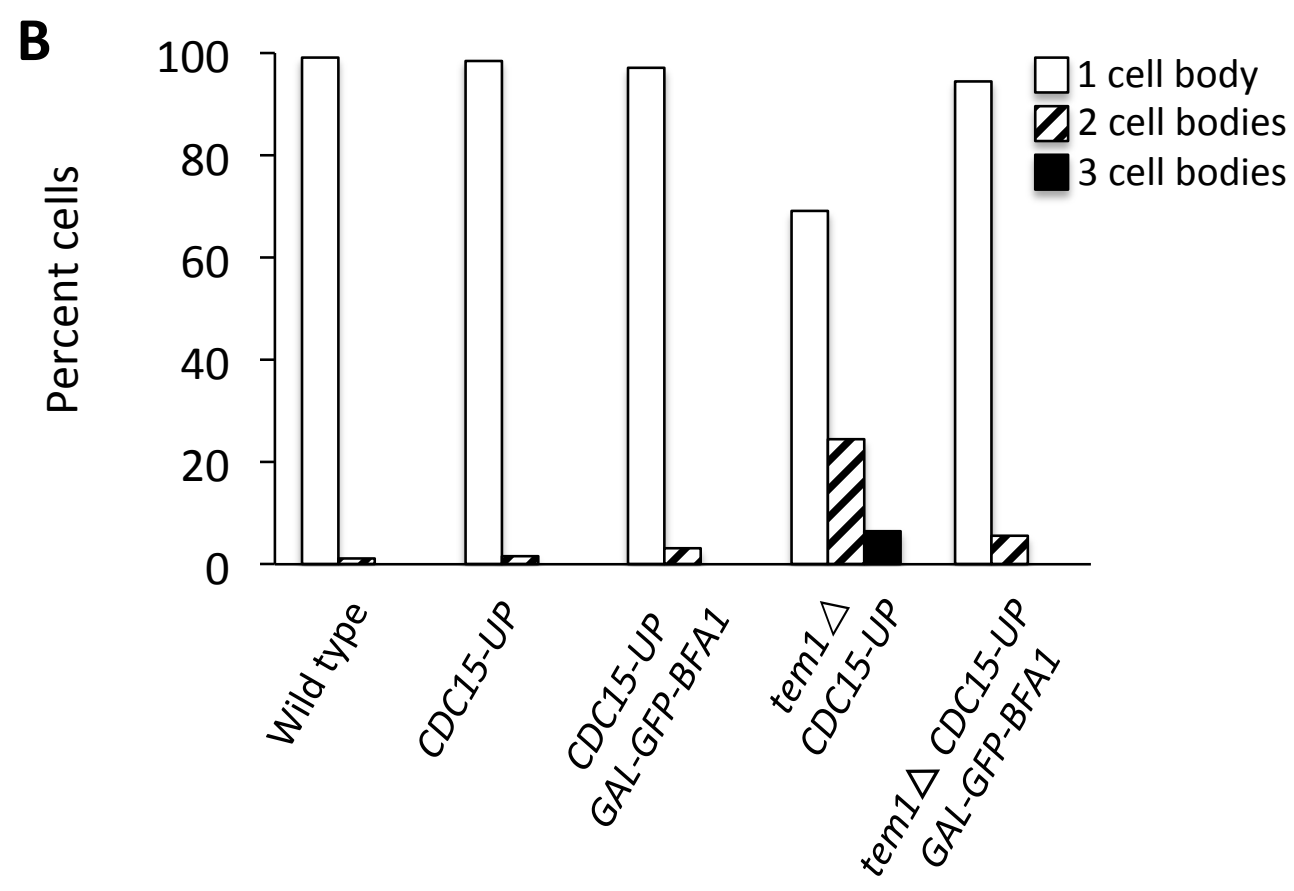

C

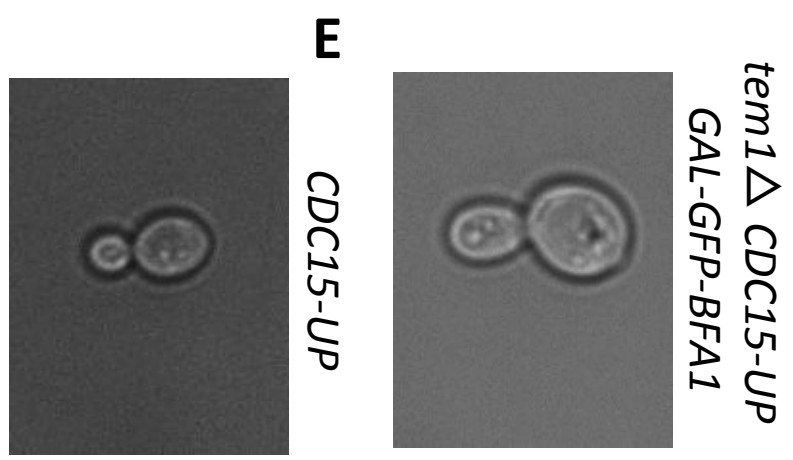

D

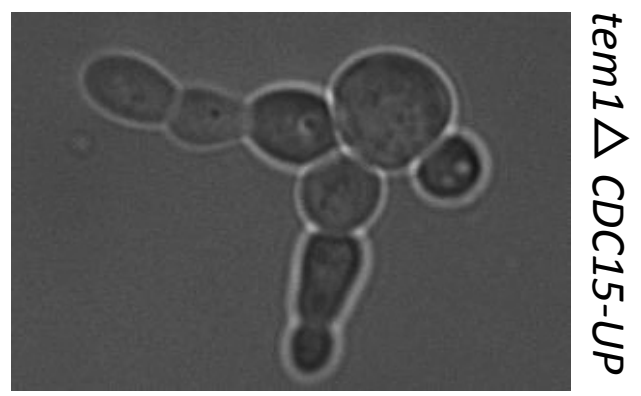

